\title{
Application of Carbohydrate-Templated Asymmetric Diels-Alder Reaction to the Syntheses of ent-Penicillones A and B
}

\author{
Chia-Hao Weng, ${ }^{\dagger}$ Day-Shin $\mathrm{Hsu}^{*} \stackrel{+}{\dagger}$ and Chun-Chen Liao ${ }^{*}, \uparrow$, \\ ${ }^{\dagger}$ Department of Chemistry, National Tsing Hua University, Hsinchu, Taiwan 300 \\ ${ }^{\ddagger}$ Department of Chemistry and Biochemistry, National Chung Cheng University, Minhsiung, Taiwan \\ 621 \\ ${ }^{\S}$ Department of Chemistry, Chung Yuan Christian University, Chungli, Taiwan 320 \\ E-mail: chedsh@ccu.edu.tw \\ ccliao@mx.nthu.edu.tw

\section{Table of Contents}

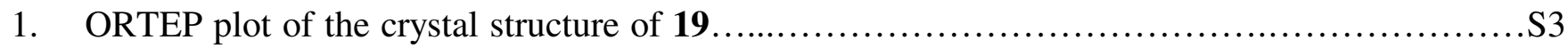

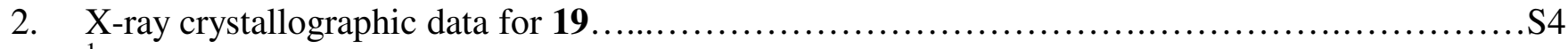

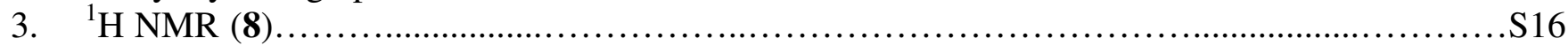

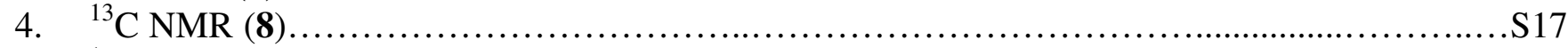

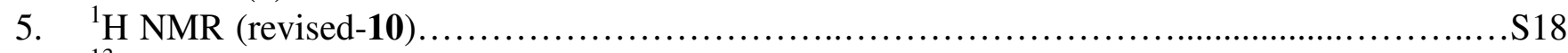

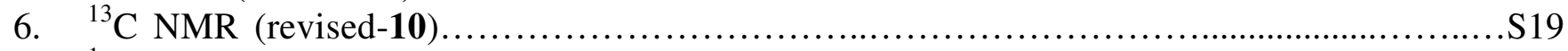

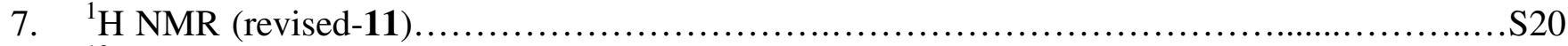

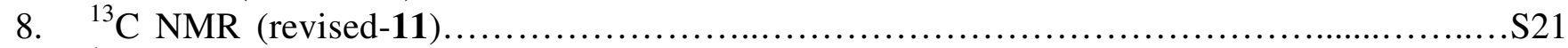

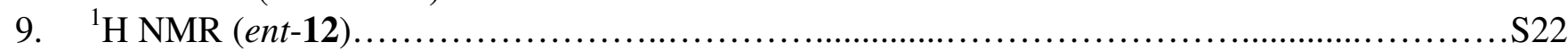

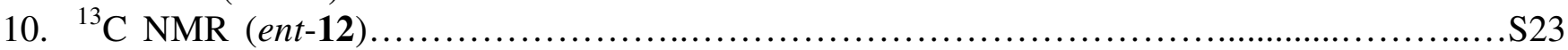

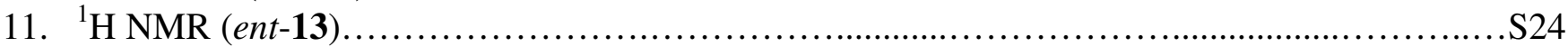

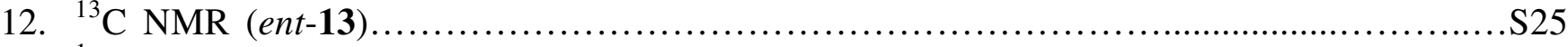

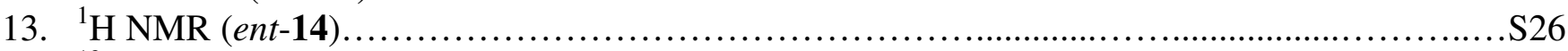

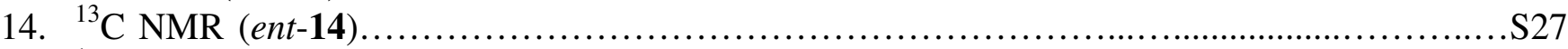

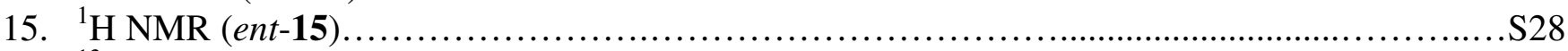

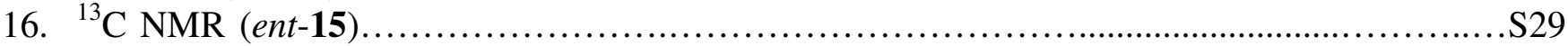

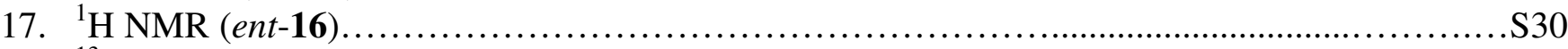

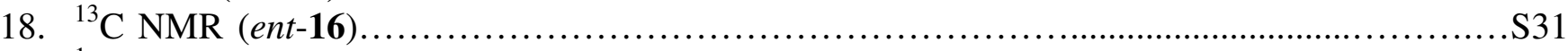

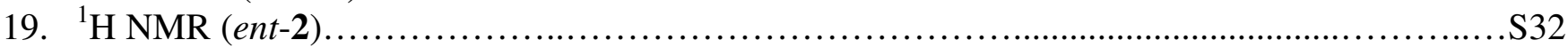

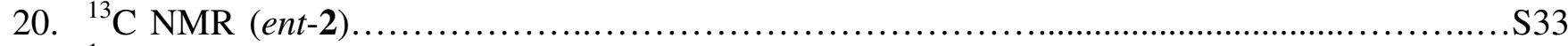

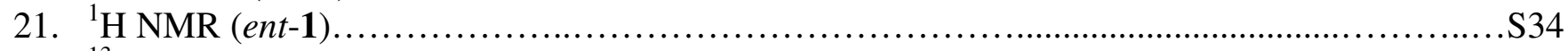

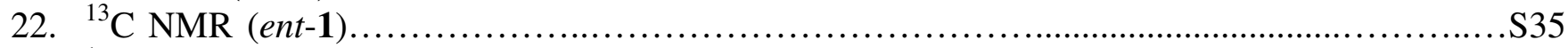

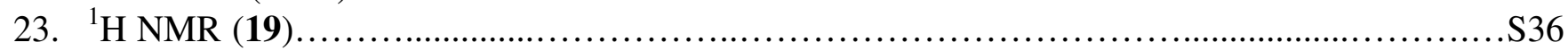




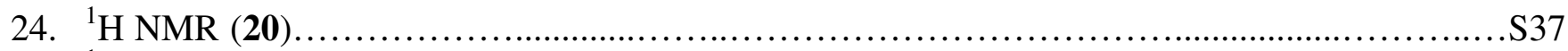

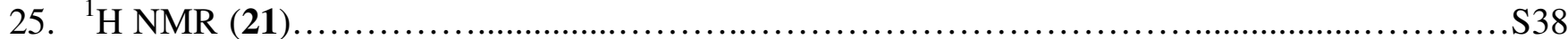

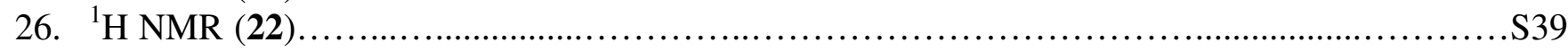




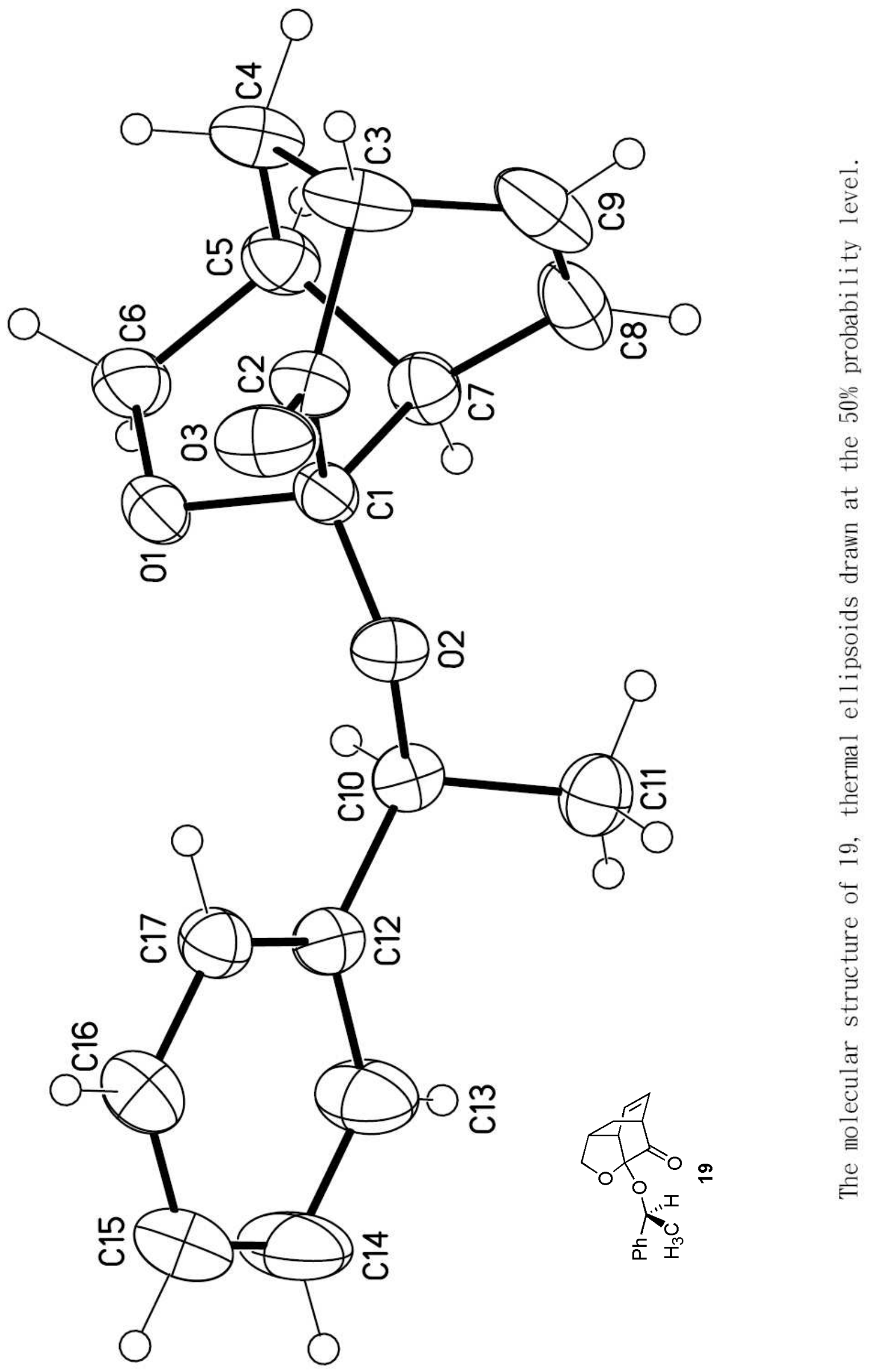




\section{X-ray crystallographic data for 19}

Table 1. Crystal data and structure refinement for $090510 \mathrm{~m}$.

Identification code

Empirical formula

Formula weight

Temperature

Wavelength

Crystal system

Space group

Unit cell dimensions

Volume

$\mathrm{Z}$

Density (calculated)

Absorption coefficient

$\mathrm{F}(000)$

Crystal size

Theta range for data collection

Index ranges

Reflections collected

Independent reflections

Completeness to theta $=28.31^{\circ}$

Absorption correction

Max. and min. transmission
$090510 \mathrm{~m}$

C17 H18 O3

270.31

295(2) K

$0.71073 \AA$

Orthorhombic

P2(1)2(1)2(1)

$\begin{array}{ll}\mathrm{a}=8.4545(9) \AA & \cdot=90^{\circ} . \\ \mathrm{b}=11.2672(12) \AA & \cdot=90^{\circ} . \\ \mathrm{c}=14.7983(15) \AA & \cdot=90^{\circ} .\end{array}$

$1409.7(3) \AA^{3}$

4

$1.274 \mathrm{Mg} / \mathrm{m}^{3}$

$0.086 \mathrm{~mm}^{-1}$

576

$0.20 \times 0.10 \times 0.10 \mathrm{~mm}^{3}$

2.27 to $28.31^{\circ}$.

$-11 \leq \mathrm{h} \leq 10,-11 \leq \mathrm{k} \leq 15,-19 \leq \mathrm{l} \leq 19$

10562

$3494[\mathrm{R}($ int $)=0.0323]$

$99.9 \%$

Empirical

0.7454 and 0.5460 
Refinement method

Data / restraints / parameters

Goodness-of-fit on $\mathrm{F}^{2}$

Final $\mathrm{R}$ indices [I $>2 \operatorname{sigma}(\mathrm{I})]$

$\mathrm{R}$ indices (all data)

Absolute structure parameter

Extinction coefficient

Largest diff. peak and hole
Full-matrix least-squares on $\mathrm{F}^{2}$

3494 / 0 / 183

1.029

$\mathrm{R} 1=0.0432, \mathrm{wR} 2=0.0947$

$\mathrm{R} 1=0.0671, \mathrm{wR} 2=0.1056$

$0.6(12)$

$0.012(2)$

0.133 and -0.131 e. $\AA^{-3}$ 
Table 2. Atomic coordinates ( $\left.\mathrm{x} 10^{4}\right)$ and equivalent isotropic displacement parameters $\left(\AA^{2} \times 10^{3}\right)$ for $090510 \mathrm{~m} . \mathrm{U}(\mathrm{eq})$ is defined as one third of the trace of the orthogonalized $\mathrm{U}^{\mathrm{ij}}$ tensor.

\begin{tabular}{|c|c|c|c|c|}
\hline & $\mathrm{x}$ & $\mathrm{y}$ & $\mathrm{z}$ & $\mathrm{U}(\mathrm{eq})$ \\
\hline $\mathrm{C}(1)$ & $3117(2)$ & $1794(1)$ & 1741(1) & $46(1)$ \\
\hline $\mathrm{C}(2)$ & $1774(2)$ & $1430(2)$ & $2379(1)$ & $56(1)$ \\
\hline $\mathrm{C}(3)$ & $2065(2)$ & 204(2) & $2778(2)$ & $75(1)$ \\
\hline $\mathrm{C}(4)$ & $3764(2)$ & $177(2)$ & $3177(2)$ & $69(1)$ \\
\hline $\mathrm{C}(5)$ & 4961(2) & $598(2)$ & $2475(1)$ & $58(1)$ \\
\hline $\mathrm{C}(6)$ & $5418(2)$ & 1891(2) & $2612(1)$ & $67(1)$ \\
\hline$C(7)$ & $4128(2)$ & $688(2)$ & $1546(1)$ & $54(1)$ \\
\hline $\mathrm{C}(8)$ & $3107(3)$ & $-349(2)$ & $1349(2)$ & $74(1)$ \\
\hline $\mathrm{C}(9)$ & $2050(3)$ & $-597(2)$ & 1963(2) & $88(1)$ \\
\hline$C(10)$ & $3480(2)$ & $2875(2)$ & $352(1)$ & $49(1)$ \\
\hline $\mathrm{C}(11)$ & $3406(2)$ & $2106(2)$ & $-484(1)$ & $63(1)$ \\
\hline$C(12)$ & $3002(2)$ & $4153(2)$ & 187(1) & $51(1)$ \\
\hline$C(13)$ & $3490(3)$ & $4746(2)$ & $-585(2)$ & $88(1)$ \\
\hline$C(14)$ & $3061(4)$ & $5906(2)$ & $-741(2)$ & $105(1)$ \\
\hline$C(15)$ & 2161(3) & $6504(2)$ & $-139(2)$ & $86(1)$ \\
\hline$C(16)$ & $1666(3)$ & $5940(2)$ & $635(2)$ & $73(1)$ \\
\hline$C(17)$ & 2081(2) & $4776(2)$ & 789(1) & $57(1)$ \\
\hline $\mathrm{O}(1)$ & $4116(2)$ & 2571(1) & $2254(1)$ & $61(1)$ \\
\hline $\mathrm{O}(2)$ & $2435(1)$ & $2390(1)$ & $1026(1)$ & $51(1)$ \\
\hline $\mathrm{O}(3)$ & $644(2)$ & $2045(1)$ & $2533(1)$ & $74(1)$ \\
\hline
\end{tabular}


Table 3. Bond lengths $[\AA]$ and angles $\left[{ }^{\circ}\right]$ for $090510 \mathrm{~m}$.

\begin{tabular}{|c|c|}
\hline $\mathrm{C}(1)-\mathrm{O}(2)$ & $1.3791(19)$ \\
\hline $\mathrm{C}(1)-\mathrm{O}(1)$ & $1.4343(19)$ \\
\hline $\mathrm{C}(1)-\mathrm{C}(2)$ & $1.533(2)$ \\
\hline $\mathrm{C}(1)-\mathrm{C}(7)$ & $1.539(2)$ \\
\hline $\mathrm{C}(2)-\mathrm{O}(3)$ & $1.202(2)$ \\
\hline$C(2)-C(3)$ & $1.523(3)$ \\
\hline $\mathrm{C}(3)-\mathrm{C}(9)$ & $1.506(4)$ \\
\hline$C(3)-C(4)$ & $1.552(3)$ \\
\hline $\mathrm{C}(3)-\mathrm{H}(3)$ & 0.9800 \\
\hline$C(4)-C(5)$ & $1.525(3)$ \\
\hline $\mathrm{C}(4)-\mathrm{H}(4 \mathrm{~A})$ & 0.9700 \\
\hline $\mathrm{C}(4)-\mathrm{H}(4 \mathrm{~B})$ & 0.9700 \\
\hline$C(5)-C(6)$ & $1.521(3)$ \\
\hline$C(5)-C(7)$ & $1.549(3)$ \\
\hline $\mathrm{C}(5)-\mathrm{H}(5)$ & 0.9800 \\
\hline $\mathrm{C}(6)-\mathrm{O}(1)$ & $1.442(2)$ \\
\hline $\mathrm{C}(6)-\mathrm{H}(6 \mathrm{~A})$ & 0.9700 \\
\hline $\mathrm{C}(6)-\mathrm{H}(6 \mathrm{~B})$ & 0.9700 \\
\hline$C(7)-C(8)$ & $1.481(3)$ \\
\hline $\mathrm{C}(7)-\mathrm{H}(7)$ & 0.9800 \\
\hline$C(8)-C(9)$ & $1.305(3)$ \\
\hline $\mathrm{C}(8)-\mathrm{H}(8)$ & 0.9300 \\
\hline $\mathrm{C}(9)-\mathrm{H}(9)$ & 0.9300 \\
\hline
\end{tabular}




\begin{tabular}{|c|c|}
\hline $\mathrm{C}(10)-\mathrm{O}(2)$ & $1.4399(19$ \\
\hline $\mathrm{C}(10)-\mathrm{C}(11)$ & $1.512(2)$ \\
\hline$C(10)-C(12)$ & $1.515(2)$ \\
\hline $\mathrm{C}(10)-\mathrm{H}(10)$ & 0.9800 \\
\hline $\mathrm{C}(11)-\mathrm{H}(11 \mathrm{~A})$ & 0.9600 \\
\hline $\mathrm{C}(11)-\mathrm{H}(11 \mathrm{~B})$ & 0.9600 \\
\hline $\mathrm{C}(11)-\mathrm{H}(11 \mathrm{C})$ & 0.9600 \\
\hline $\mathrm{C}(12)-\mathrm{C}(17)$ & $1.376(3)$ \\
\hline$C(12)-C(13)$ & $1.386(3)$ \\
\hline $\mathrm{C}(13)-\mathrm{C}(14)$ & $1.377(3)$ \\
\hline $\mathrm{C}(13)-\mathrm{H}(13)$ & 0.9300 \\
\hline $\mathrm{C}(14)-\mathrm{C}(15)$ & $1.351(4)$ \\
\hline $\mathrm{C}(14)-\mathrm{H}(14)$ & 0.9300 \\
\hline$C(15)-C(16)$ & $1.375(3)$ \\
\hline $\mathrm{C}(15)-\mathrm{H}(15)$ & 0.9300 \\
\hline$C(16)-C(17)$ & $1.377(3)$ \\
\hline $\mathrm{C}(16)-\mathrm{H}(16)$ & 0.9300 \\
\hline $\mathrm{C}(17)-\mathrm{H}(17)$ & 0.9300 \\
\hline $\mathrm{O}(2)-\mathrm{C}(1)-\mathrm{O}(1)$ & $110.80(12)$ \\
\hline $\mathrm{O}(2)-\mathrm{C}(1)-\mathrm{C}(2)$ & $107.03(13)$ \\
\hline $\mathrm{O}(1)-\mathrm{C}(1)-\mathrm{C}(2)$ & $105.83(13)$ \\
\hline $\mathrm{O}(2)-\mathrm{C}(1)-\mathrm{C}(7)$ & $118.87(13)$ \\
\hline $\mathrm{O}(1)-\mathrm{C}(1)-\mathrm{C}(7)$ & $105.52(13)$ \\
\hline$C(2)-C(1)-C(7)$ & $108.08(14)$ \\
\hline
\end{tabular}




\begin{tabular}{|c|c|}
\hline $\mathrm{O}(3)-\mathrm{C}(2)-\mathrm{C}(3)$ & $125.29(17)$ \\
\hline $\mathrm{O}(3)-\mathrm{C}(2)-\mathrm{C}(1)$ & $123.50(16)$ \\
\hline$C(3)-C(2)-C(1)$ & $111.21(15)$ \\
\hline $\mathrm{C}(9)-\mathrm{C}(3)-\mathrm{C}(2)$ & $103.40(17)$ \\
\hline$C(9)-C(3)-C(4)$ & $107.49(18)$ \\
\hline$C(2)-C(3)-C(4)$ & $108.33(17)$ \\
\hline $\mathrm{C}(9)-\mathrm{C}(3)-\mathrm{H}(3)$ & 112.4 \\
\hline $\mathrm{C}(2)-\mathrm{C}(3)-\mathrm{H}(3)$ & 112.4 \\
\hline $\mathrm{C}(4)-\mathrm{C}(3)-\mathrm{H}(3)$ & 112.4 \\
\hline$C(5)-C(4)-C(3)$ & $110.44(16)$ \\
\hline $\mathrm{C}(5)-\mathrm{C}(4)-\mathrm{H}(4 \mathrm{~A})$ & 109.6 \\
\hline $\mathrm{C}(3)-\mathrm{C}(4)-\mathrm{H}(4 \mathrm{~A})$ & 109.6 \\
\hline $\mathrm{C}(5)-\mathrm{C}(4)-\mathrm{H}(4 \mathrm{~B})$ & 109.6 \\
\hline $\mathrm{C}(3)-\mathrm{C}(4)-\mathrm{H}(4 \mathrm{~B})$ & 109.6 \\
\hline $\mathrm{H}(4 \mathrm{~A})-\mathrm{C}(4)-\mathrm{H}(4 \mathrm{~B})$ & 108.1 \\
\hline$C(6)-C(5)-C(4)$ & $112.08(17)$ \\
\hline$C(6)-C(5)-C(7)$ & $99.84(13)$ \\
\hline$C(4)-C(5)-C(7)$ & $108.85(16)$ \\
\hline $\mathrm{C}(6)-\mathrm{C}(5)-\mathrm{H}(5)$ & 111.8 \\
\hline $\mathrm{C}(4)-\mathrm{C}(5)-\mathrm{H}(5)$ & 111.8 \\
\hline $\mathrm{C}(7)-\mathrm{C}(5)-\mathrm{H}(5)$ & 111.8 \\
\hline $\mathrm{O}(1)-\mathrm{C}(6)-\mathrm{C}(5)$ & $105.46(14)$ \\
\hline $\mathrm{O}(1)-\mathrm{C}(6)-\mathrm{H}(6 \mathrm{~A})$ & 110.6 \\
\hline $\mathrm{C}(5)-\mathrm{C}(6)-\mathrm{H}(6 \mathrm{~A})$ & 110.6 \\
\hline $\mathrm{O}(1)-\mathrm{C}(6)-\mathrm{H}(6 \mathrm{~B})$ & 110.6 \\
\hline
\end{tabular}




\begin{tabular}{|c|c|}
\hline $\mathrm{C}(5)-\mathrm{C}(6)-\mathrm{H}(6 \mathrm{~B})$ & 110.6 \\
\hline $\mathrm{H}(6 \mathrm{~A})-\mathrm{C}(6)-\mathrm{H}(6 \mathrm{~B})$ & 108.8 \\
\hline $\mathrm{C}(8)-\mathrm{C}(7)-\mathrm{C}(1)$ & $110.61(15)$ \\
\hline$C(8)-C(7)-C(5)$ & $112.80(16)$ \\
\hline$C(1)-C(7)-C(5)$ & $97.98(13)$ \\
\hline $\mathrm{C}(8)-\mathrm{C}(7)-\mathrm{H}(7)$ & 111.6 \\
\hline $\mathrm{C}(1)-\mathrm{C}(7)-\mathrm{H}(7)$ & 111.6 \\
\hline $\mathrm{C}(5)-\mathrm{C}(7)-\mathrm{H}(7)$ & 111.6 \\
\hline$C(9)-C(8)-C(7)$ & $115.6(2)$ \\
\hline $\mathrm{C}(9)-\mathrm{C}(8)-\mathrm{H}(8)$ & 122.2 \\
\hline $\mathrm{C}(7)-\mathrm{C}(8)-\mathrm{H}(8)$ & 122.2 \\
\hline $\mathrm{C}(8)-\mathrm{C}(9)-\mathrm{C}(3)$ & $115.01(19)$ \\
\hline $\mathrm{C}(8)-\mathrm{C}(9)-\mathrm{H}(9)$ & 122.5 \\
\hline $\mathrm{C}(3)-\mathrm{C}(9)-\mathrm{H}(9)$ & 122.5 \\
\hline $\mathrm{O}(2)-\mathrm{C}(10)-\mathrm{C}(11)$ & $108.92(14)$ \\
\hline $\mathrm{O}(2)-\mathrm{C}(10)-\mathrm{C}(12)$ & $107.96(14)$ \\
\hline $\mathrm{C}(11)-\mathrm{C}(10)-\mathrm{C}(12)$ & $113.69(14)$ \\
\hline $\mathrm{O}(2)-\mathrm{C}(10)-\mathrm{H}(10)$ & 108.7 \\
\hline $\mathrm{C}(11)-\mathrm{C}(10)-\mathrm{H}(10)$ & 108.7 \\
\hline $\mathrm{C}(12)-\mathrm{C}(10)-\mathrm{H}(10)$ & 108.7 \\
\hline $\mathrm{C}(10)-\mathrm{C}(11)-\mathrm{H}(11 \mathrm{~A})$ & 109.5 \\
\hline $\mathrm{C}(10)-\mathrm{C}(11)-\mathrm{H}(11 \mathrm{~B})$ & 109.5 \\
\hline $\mathrm{H}(11 \mathrm{~A})-\mathrm{C}(11)-\mathrm{H}(11 \mathrm{~B})$ & 109.5 \\
\hline $\mathrm{C}(10)-\mathrm{C}(11)-\mathrm{H}(11 \mathrm{C})$ & 109.5 \\
\hline $\mathrm{H}(11 \mathrm{~A})-\mathrm{C}(11)-\mathrm{H}(11 \mathrm{C})$ & 109.5 \\
\hline
\end{tabular}




\begin{tabular}{|c|c|}
\hline $\mathrm{H}(11 \mathrm{~B})-\mathrm{C}(11)-\mathrm{H}(11 \mathrm{C})$ & 109.5 \\
\hline $\mathrm{C}(17)-\mathrm{C}(12)-\mathrm{C}(13)$ & $117.15(18)$ \\
\hline $\mathrm{C}(17)-\mathrm{C}(12)-\mathrm{C}(10)$ & $122.12(16)$ \\
\hline C(13)-C(12)-C(10) & $120.72(18)$ \\
\hline$C(14)-C(13)-C(12)$ & $121.2(2)$ \\
\hline $\mathrm{C}(14)-\mathrm{C}(13)-\mathrm{H}(13)$ & 119.4 \\
\hline $\mathrm{C}(12)-\mathrm{C}(13)-\mathrm{H}(13)$ & 119.4 \\
\hline $\mathrm{C}(15)-\mathrm{C}(14)-\mathrm{C}(13)$ & $120.8(2)$ \\
\hline $\mathrm{C}(15)-\mathrm{C}(14)-\mathrm{H}(14)$ & 119.6 \\
\hline $\mathrm{C}(13)-\mathrm{C}(14)-\mathrm{H}(14)$ & 119.6 \\
\hline$C(14)-C(15)-C(16)$ & 119.3(2) \\
\hline $\mathrm{C}(14)-\mathrm{C}(15)-\mathrm{H}(15)$ & 120.3 \\
\hline $\mathrm{C}(16)-\mathrm{C}(15)-\mathrm{H}(15)$ & 120.3 \\
\hline$C(15)-C(16)-C(17)$ & $120.1(2)$ \\
\hline $\mathrm{C}(15)-\mathrm{C}(16)-\mathrm{H}(16)$ & 120.0 \\
\hline $\mathrm{C}(17)-\mathrm{C}(16)-\mathrm{H}(16)$ & 120.0 \\
\hline $\mathrm{C}(12)-\mathrm{C}(17)-\mathrm{C}(16)$ & $121.53(18)$ \\
\hline $\mathrm{C}(12)-\mathrm{C}(17)-\mathrm{H}(17)$ & 119.2 \\
\hline $\mathrm{C}(16)-\mathrm{C}(17)-\mathrm{H}(17)$ & 119.2 \\
\hline $\mathrm{C}(1)-\mathrm{O}(1)-\mathrm{C}(6)$ & $108.58(12)$ \\
\hline $\mathrm{C}(1)-\mathrm{O}(2)-\mathrm{C}(10)$ & $117.36(12)$ \\
\hline
\end{tabular}

Symmetry transformations used to generate equivalent atoms: 
Table 4. Anisotropic displacement parameters $\left(\AA^{2} \times 10^{3}\right)$ for $090510 \mathrm{~m}$. The anisotropic displacement factor exponent takes the form: $-2 \cdot{ }^{2}\left[\mathrm{~h}^{2} \mathrm{a}^{* 2} \mathrm{U}^{11}+\ldots+2 \mathrm{~h} \mathrm{k} \mathrm{a}^{*} \mathrm{~b}^{*} \mathrm{U}^{12}\right]$

\begin{tabular}{|c|c|c|c|c|c|c|}
\hline & $\mathrm{U}^{11}$ & $\mathrm{U}^{22}$ & $\mathrm{U}^{33}$ & $\mathrm{U}^{23}$ & $\mathrm{U}^{13}$ & $\mathrm{U}^{12}$ \\
\hline $\mathrm{C}(1)$ & $50(1)$ & $37(1)$ & $51(1)$ & 1(1) & $-2(1)$ & $0(1)$ \\
\hline $\mathrm{C}(2)$ & $56(1)$ & $49(1)$ & $65(1)$ & $8(1)$ & $4(1)$ & $5(1)$ \\
\hline$C(3)$ & $59(1)$ & $61(1)$ & $106(2)$ & $38(1)$ & $16(1)$ & $7(1)$ \\
\hline$C(4)$ & 71(1) & $53(1)$ & $84(1)$ & $24(1)$ & 1(1) & $7(1)$ \\
\hline$C(5)$ & $52(1)$ & $49(1)$ & $72(1)$ & $8(1)$ & $-8(1)$ & $2(1)$ \\
\hline$C(6)$ & $70(1)$ & $57(1)$ & $73(1)$ & $12(1)$ & $-21(1)$ & $-11(1)$ \\
\hline$C(7)$ & $49(1)$ & $49(1)$ & $63(1)$ & $-2(1)$ & $-4(1)$ & $8(1)$ \\
\hline $\mathrm{C}(8)$ & $70(1)$ & $44(1)$ & $109(2)$ & $-16(1)$ & $-23(1)$ & $6(1)$ \\
\hline $\mathrm{C}(9)$ & $65(1)$ & $40(1)$ & $159(2)$ & $10(1)$ & $-29(2)$ & $-4(1)$ \\
\hline$C(10)$ & $39(1)$ & $55(1)$ & $51(1)$ & $4(1)$ & $0(1)$ & $-1(1)$ \\
\hline $\mathrm{C}(11)$ & $61(1)$ & $69(1)$ & $61(1)$ & $-7(1)$ & $0(1)$ & $4(1)$ \\
\hline $\mathrm{C}(12)$ & $45(1)$ & $55(1)$ & $53(1)$ & $6(1)$ & $-2(1)$ & $-4(1)$ \\
\hline$C(13)$ & $108(2)$ & $75(1)$ & $80(1)$ & $20(1)$ & $36(1)$ & $7(1)$ \\
\hline $\mathrm{C}(14)$ & $141(3)$ & $77(2)$ & $96(2)$ & $39(1)$ & $35(2)$ & $4(2)$ \\
\hline$C(15)$ & $99(2)$ & $55(1)$ & $105(2)$ & $20(1)$ & $4(2)$ & $3(1)$ \\
\hline$C(16)$ & $75(1)$ & $56(1)$ & $89(1)$ & $-4(1)$ & $6(1)$ & $3(1)$ \\
\hline$C(17)$ & $62(1)$ & $53(1)$ & $58(1)$ & $2(1)$ & $4(1)$ & $-4(1)$ \\
\hline $\mathrm{O}(1)$ & $74(1)$ & $40(1)$ & $69(1)$ & 1(1) & $-17(1)$ & $-6(1)$ \\
\hline $\mathrm{O}(2)$ & $43(1)$ & $53(1)$ & $56(1)$ & 11(1) & $1(1)$ & 1(1) \\
\hline $\mathrm{O}(3)$ & $72(1)$ & $73(1)$ & $75(1)$ & 19(1) & 21(1) & $24(1)$ \\
\hline
\end{tabular}


S-14 
Table 5. Hydrogen coordinates ( $\left.\times 10^{4}\right)$ and isotropic displacement parameters $\left(\AA^{2} \times 10^{3}\right)$ for $090510 \mathrm{~m}$.

\begin{tabular}{|c|c|c|c|c|}
\hline & $\mathrm{x}$ & $\mathrm{y}$ & $\mathrm{z}$ & $\mathrm{U}(\mathrm{eq})$ \\
\hline $\mathrm{H}(3)$ & 1261 & -18 & 3224 & 90 \\
\hline $\mathrm{H}(4 \mathrm{~A})$ & 3814 & 686 & 3704 & 83 \\
\hline $\mathrm{H}(4 \mathrm{~B})$ & 4022 & -625 & 3364 & 83 \\
\hline $\mathrm{H}(5)$ & 5891 & 79 & 2451 & 69 \\
\hline $\mathrm{H}(6 \mathrm{~A})$ & 5572 & 2061 & 3248 & 80 \\
\hline $\mathrm{H}(6 \mathrm{~B})$ & 6388 & 2073 & 2290 & 80 \\
\hline $\mathrm{H}(7)$ & 4887 & 832 & 1057 & 64 \\
\hline $\mathrm{H}(8)$ & 3212 & -794 & 823 & 89 \\
\hline $\mathrm{H}(9)$ & 1339 & -1223 & 1902 & 105 \\
\hline $\mathrm{H}(10)$ & 4563 & 2862 & 587 & 58 \\
\hline $\mathrm{H}(11 \mathrm{~A})$ & 2364 & 2146 & -740 & 95 \\
\hline $\mathrm{H}(11 \mathrm{~B})$ & 3645 & 1299 & -326 & 95 \\
\hline $\mathrm{H}(11 \mathrm{C})$ & 4164 & 2384 & -919 & 95 \\
\hline $\mathrm{H}(13)$ & 4119 & 4352 & -1004 & 105 \\
\hline $\mathrm{H}(14)$ & 3393 & 6283 & -1267 & 126 \\
\hline $\mathrm{H}(15)$ & 1880 & 7290 & -247 & 104 \\
\hline $\mathrm{H}(16)$ & 1049 & 6345 & 1054 & 88 \\
\hline $\mathrm{H}(17)$ & 1731 & 4402 & 1313 & 69 \\
\hline
\end{tabular}




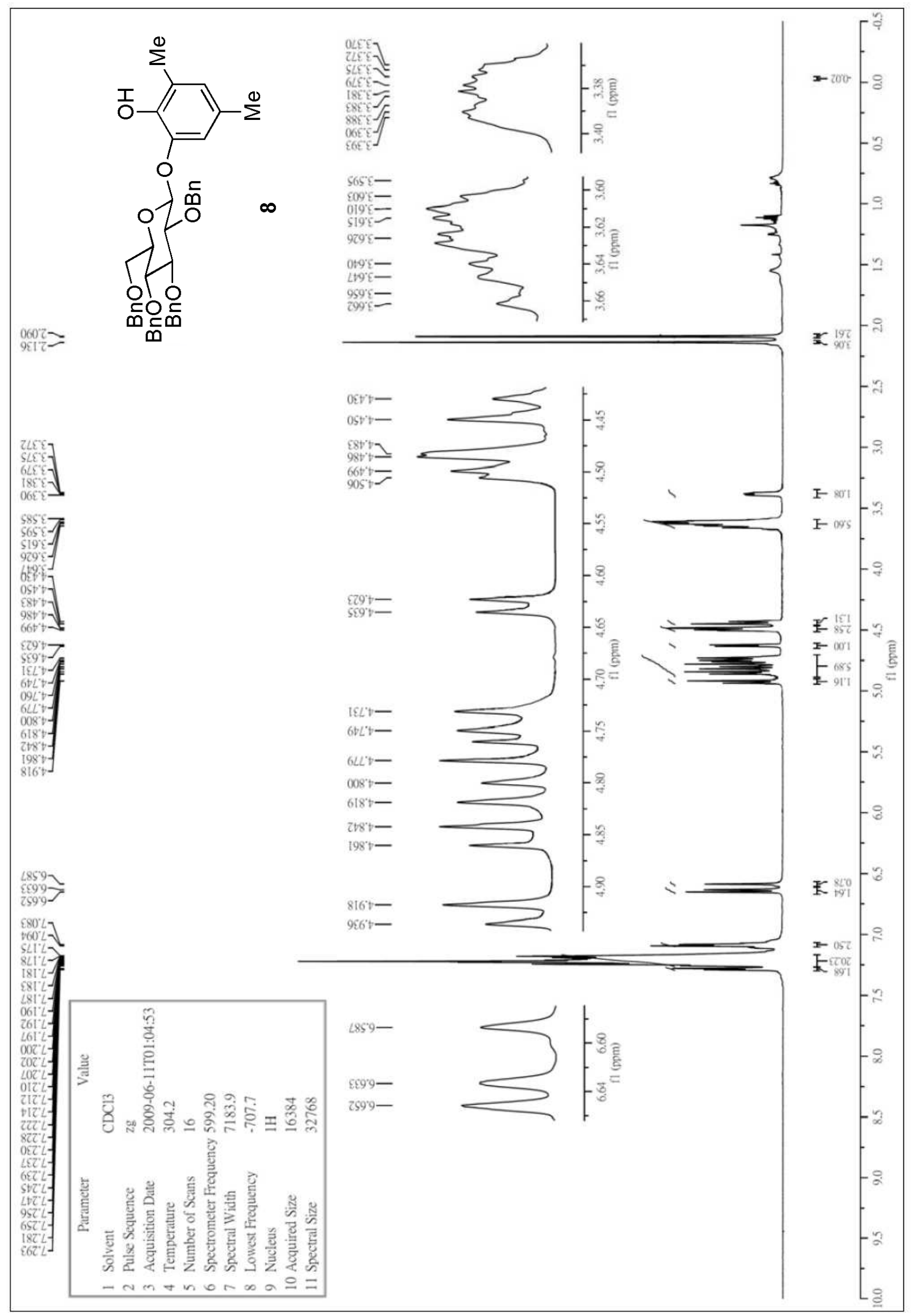




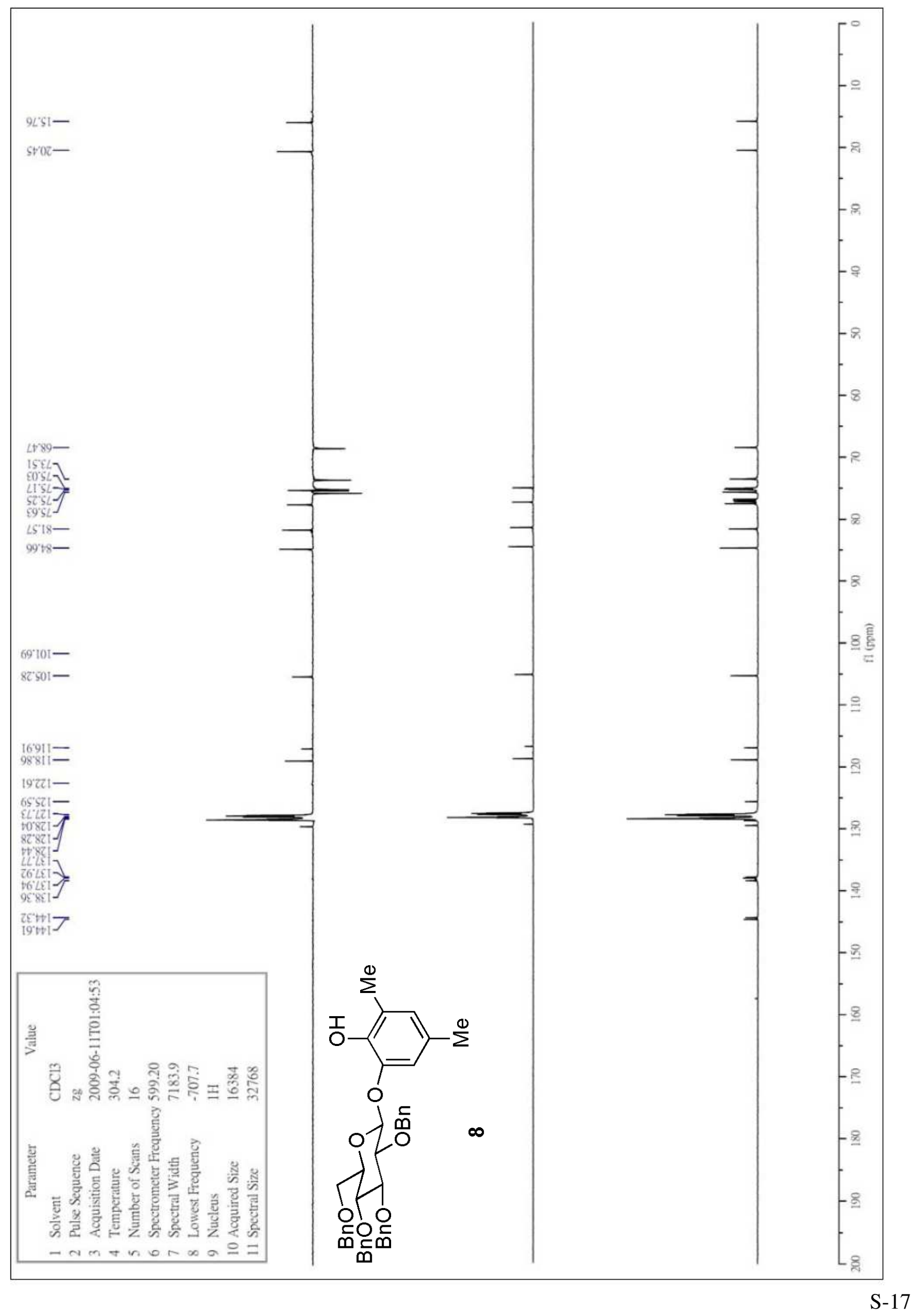




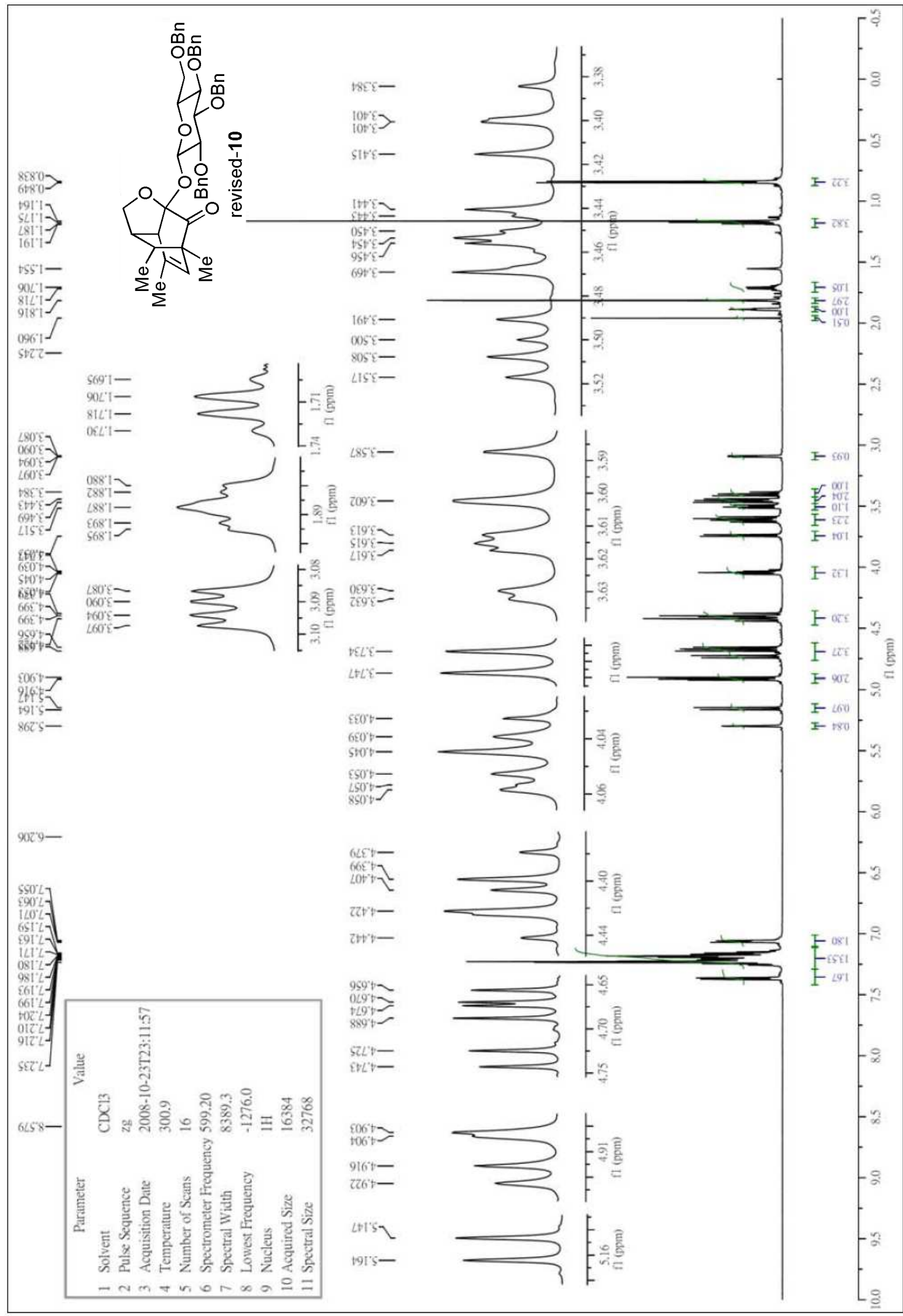




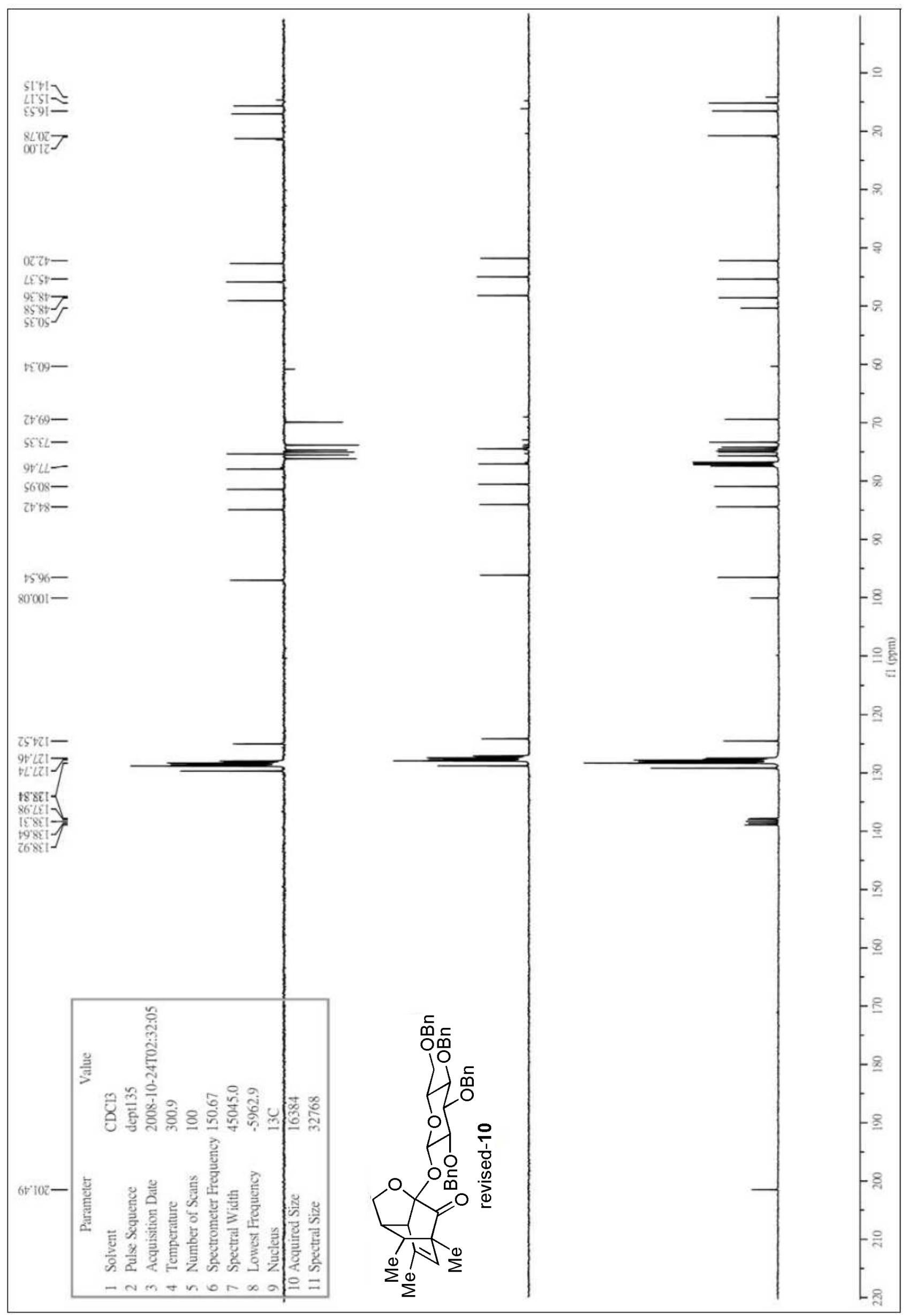




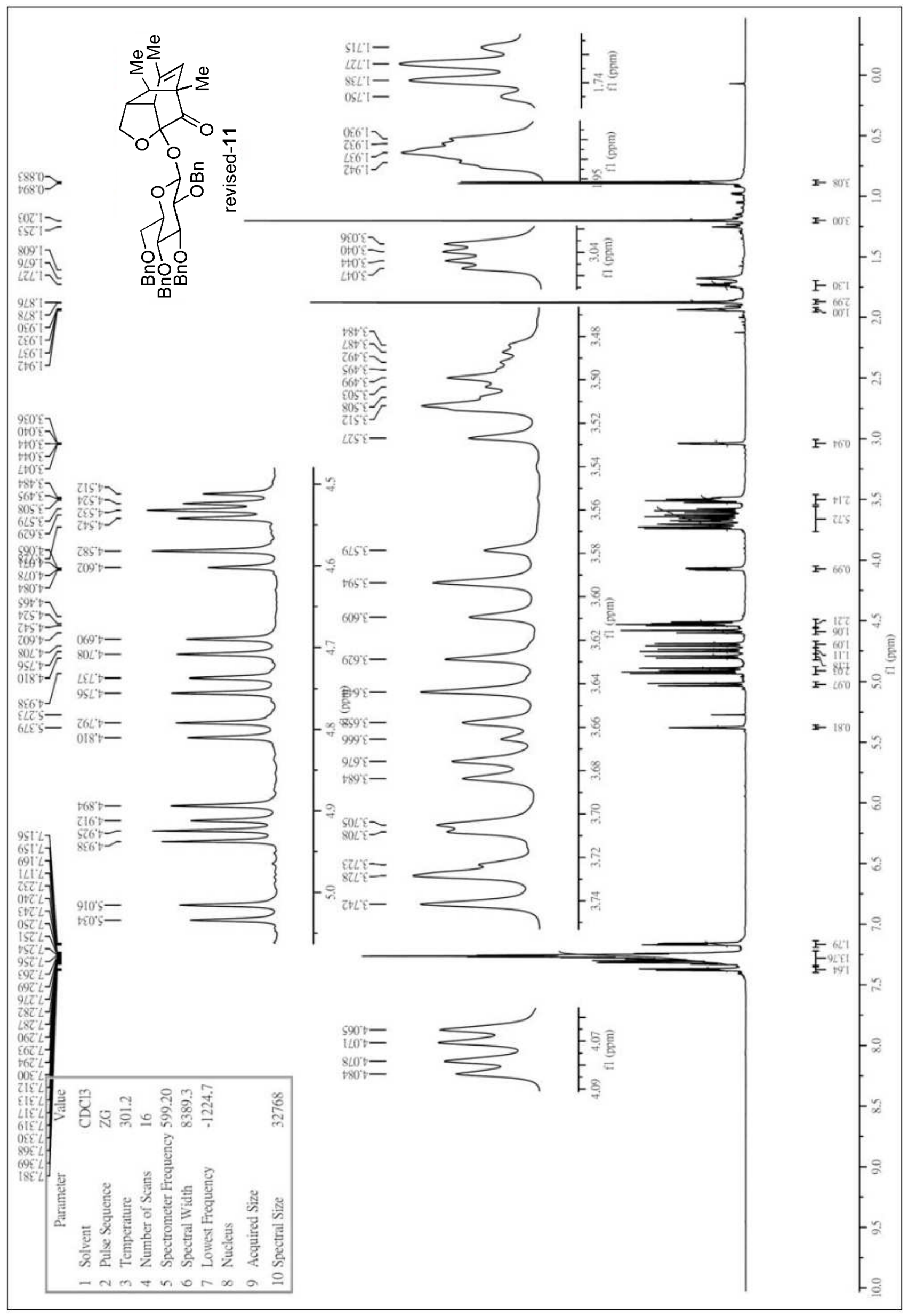




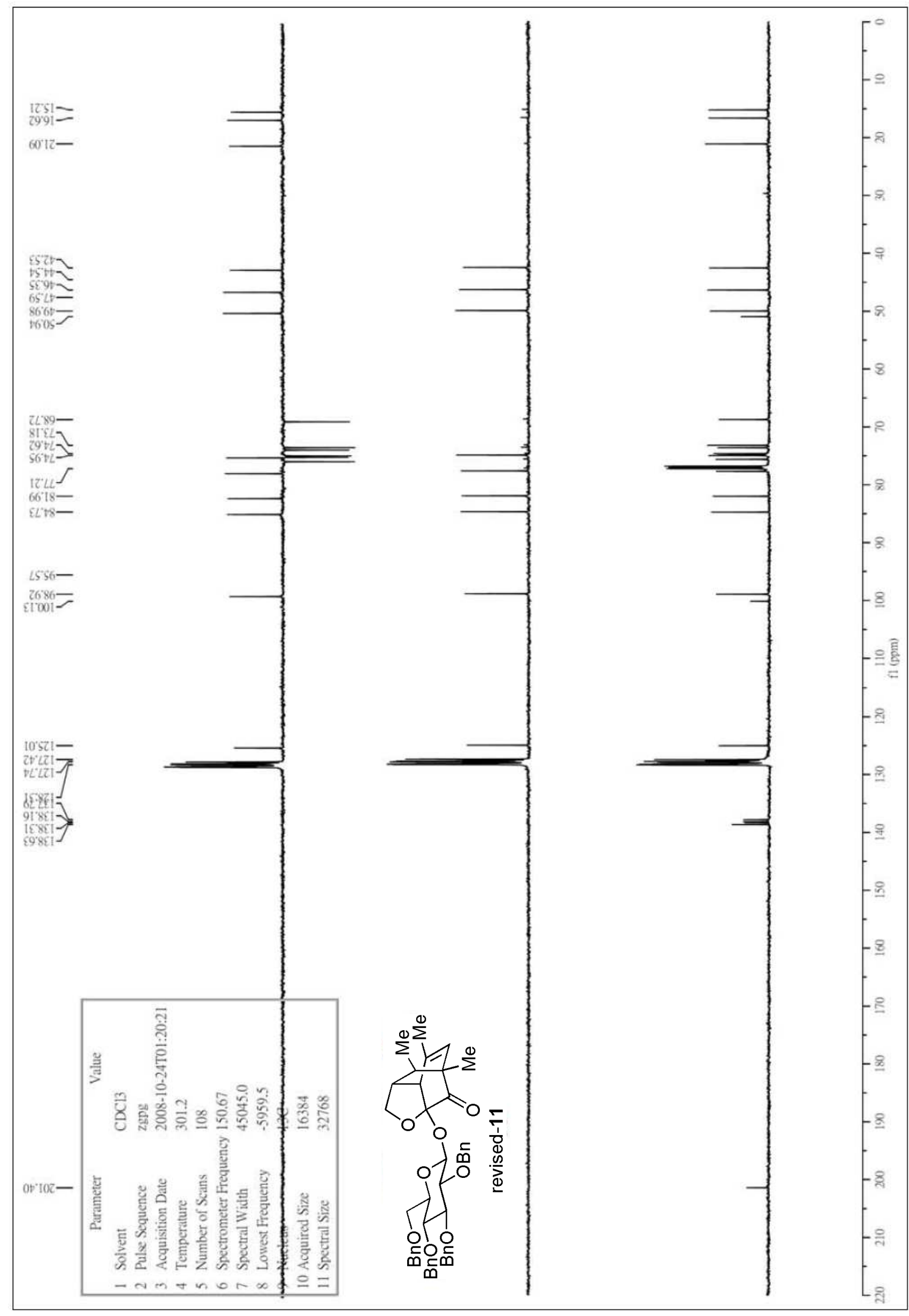




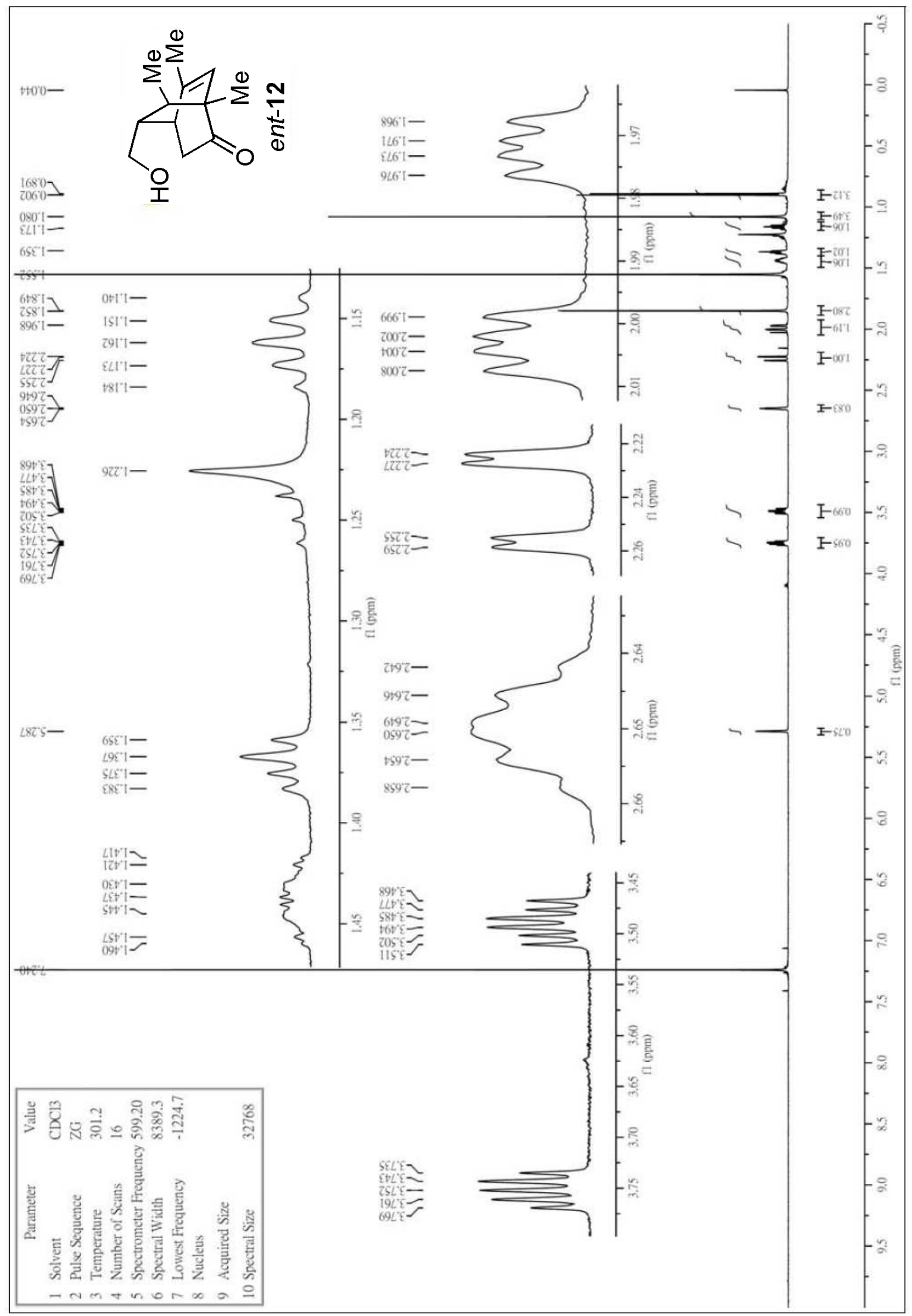




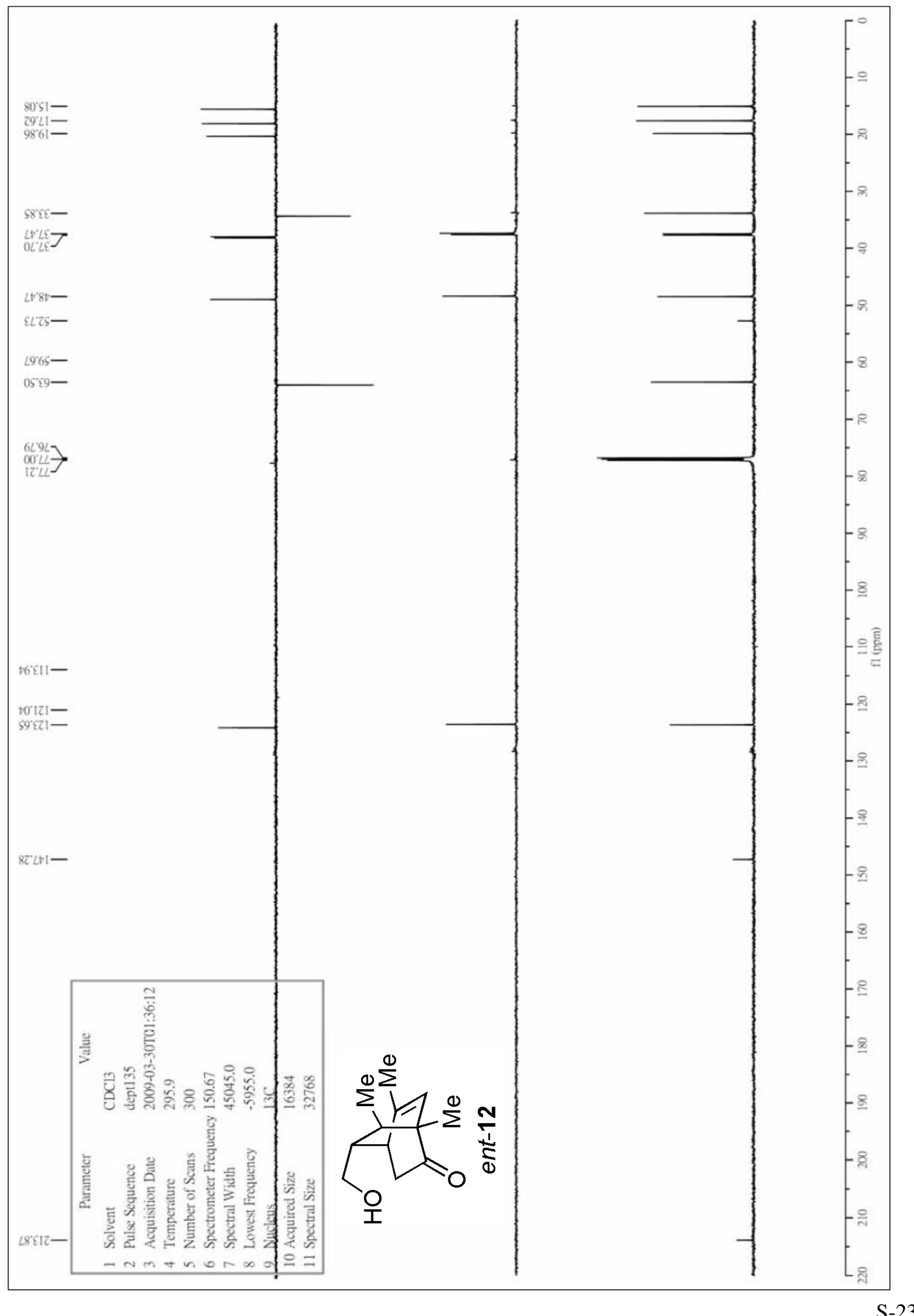




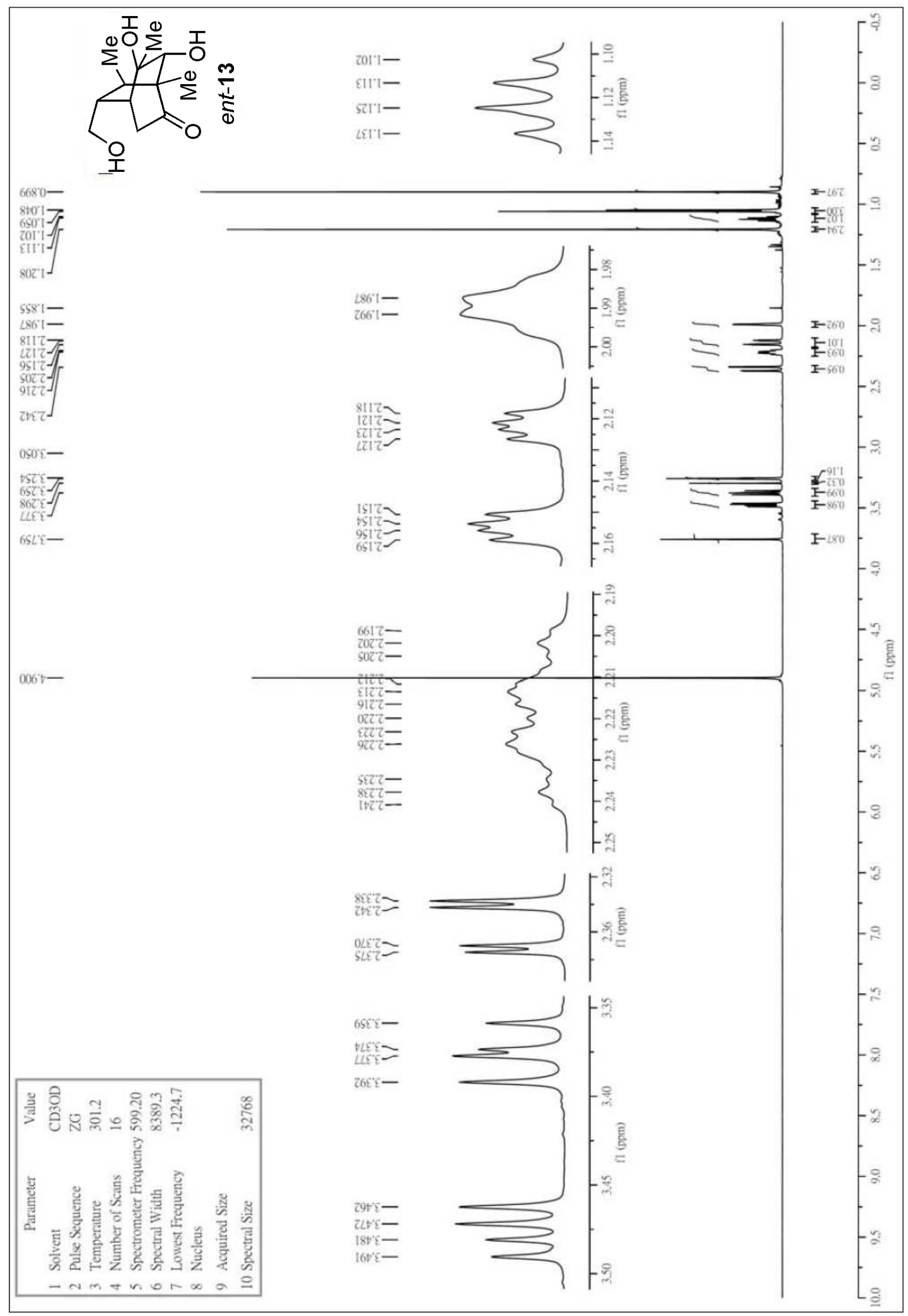




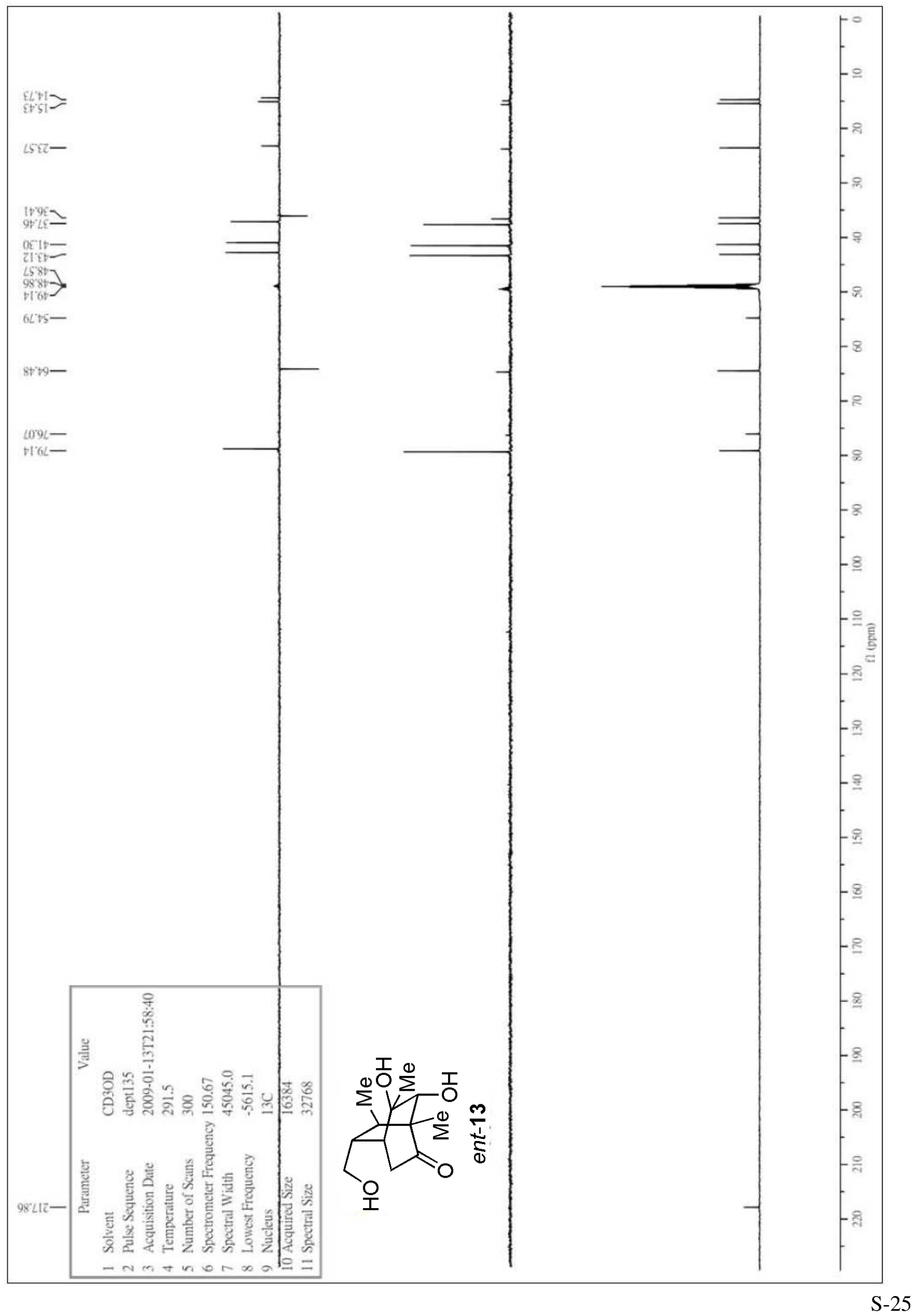




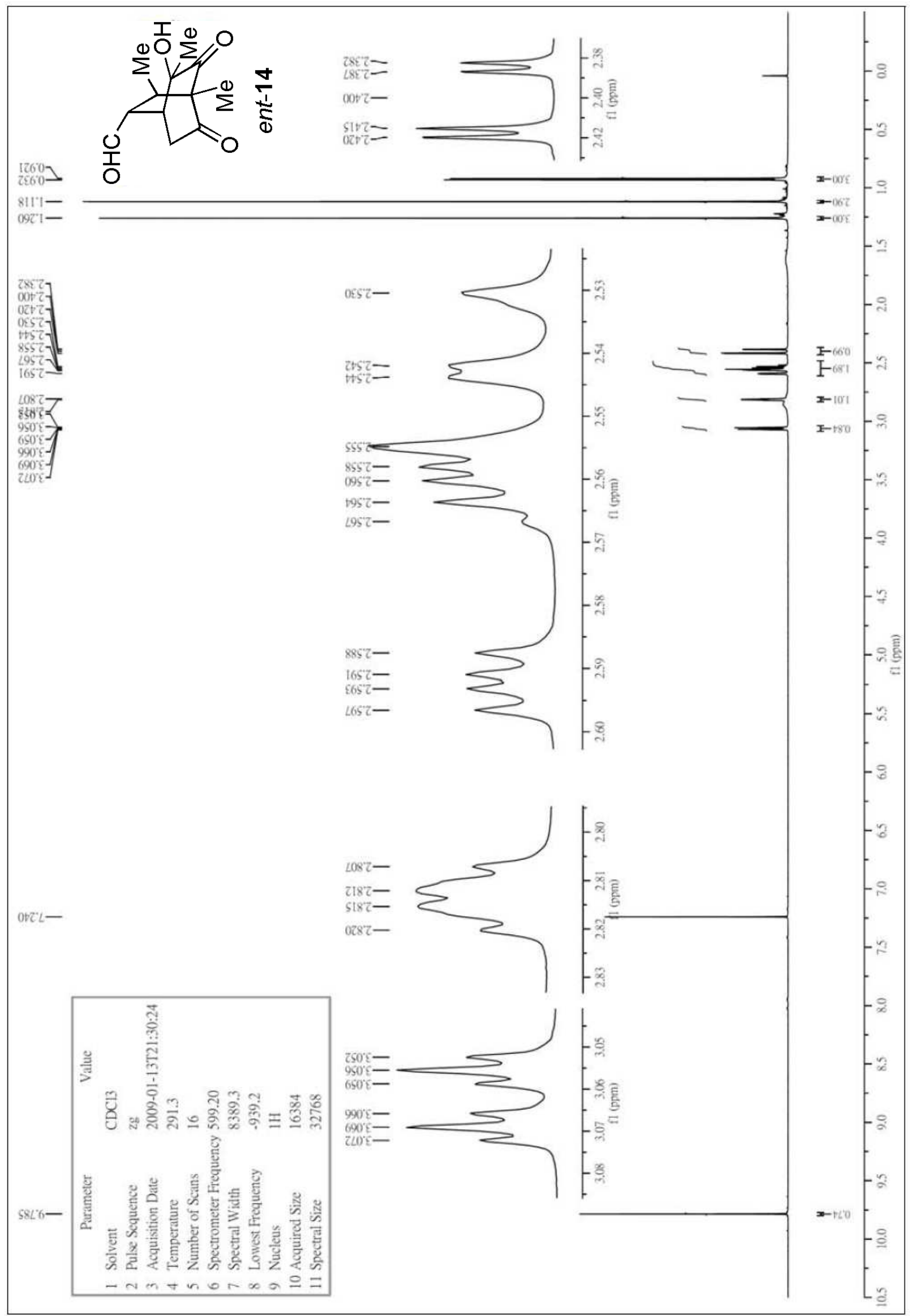




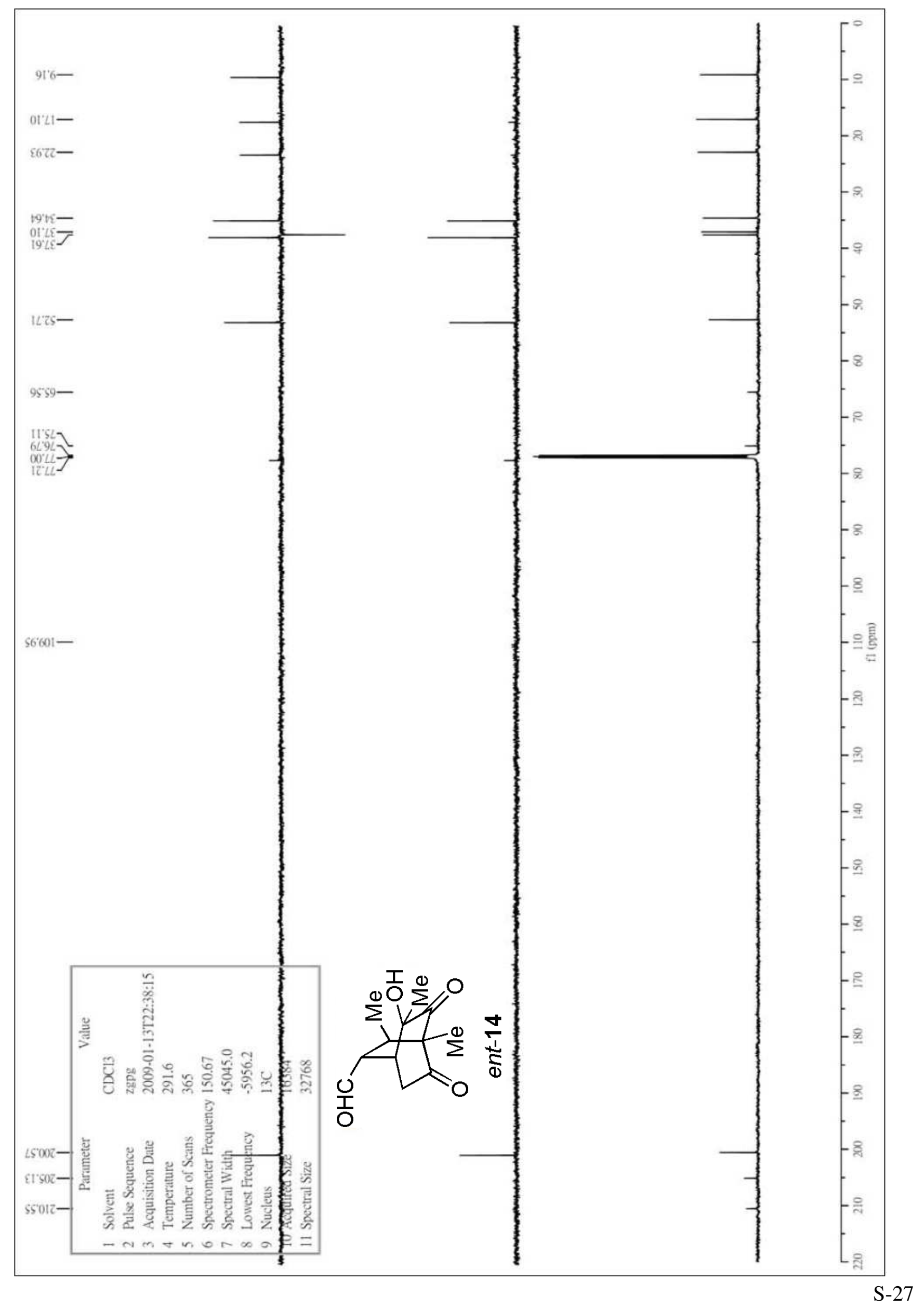




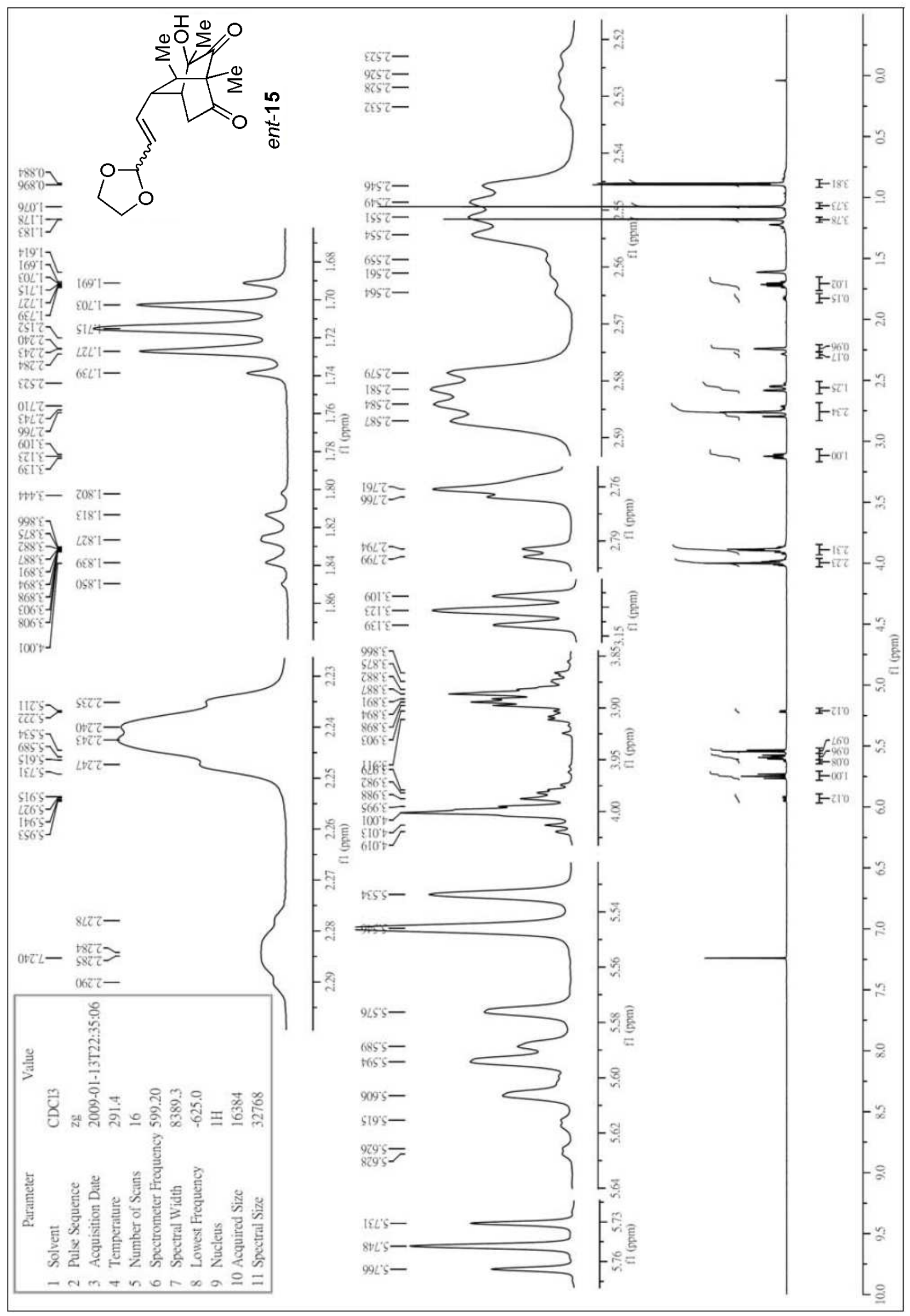




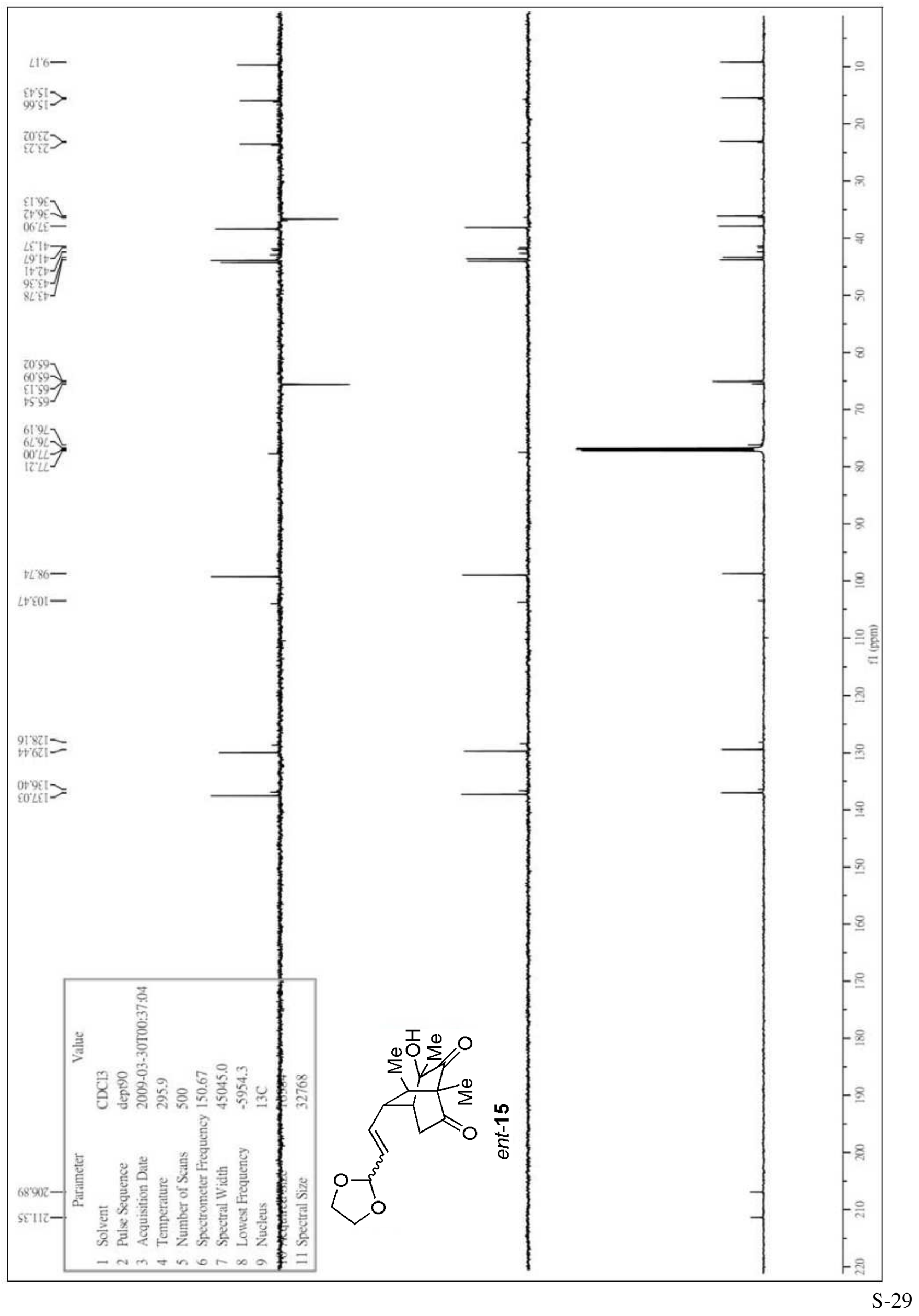




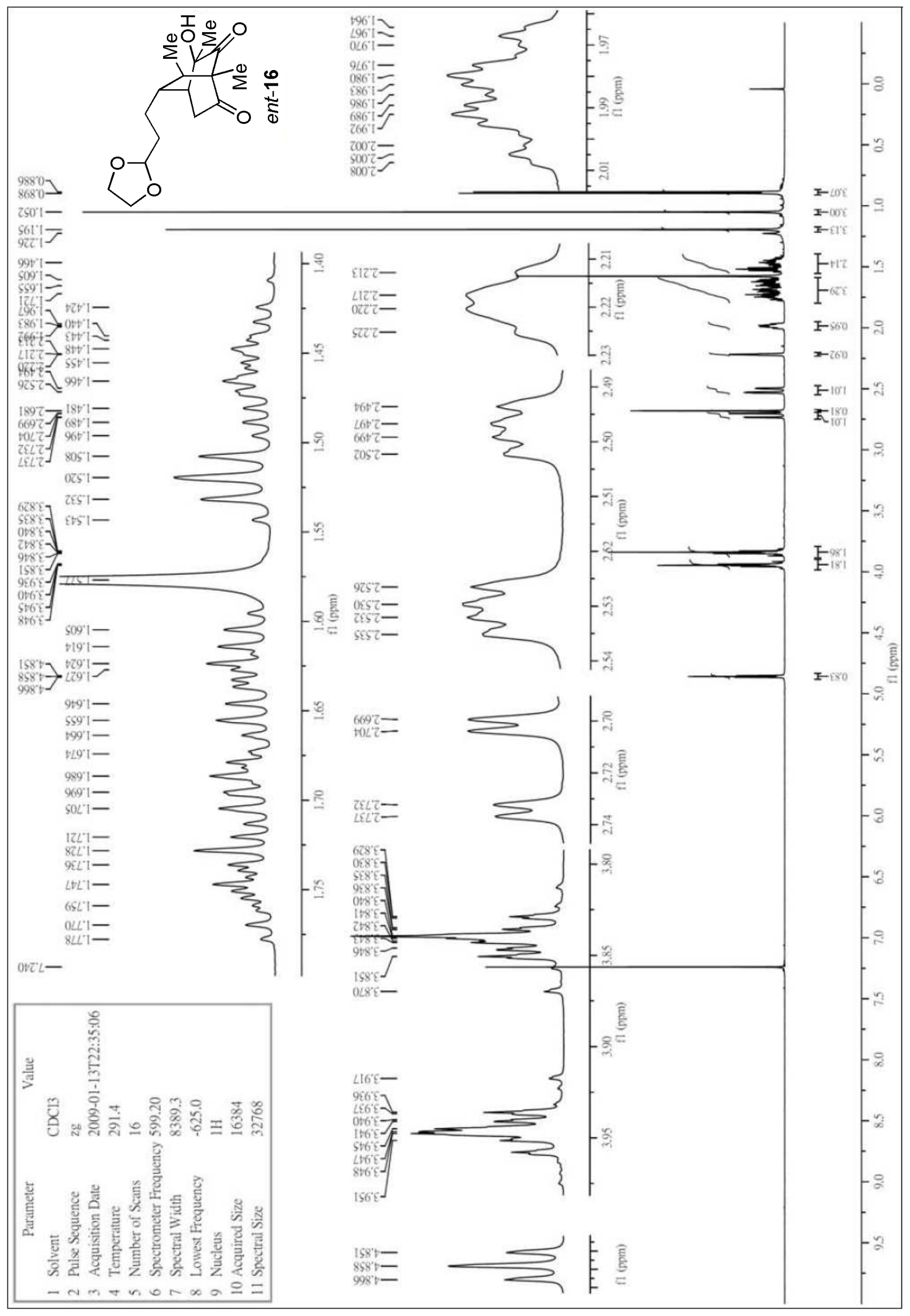




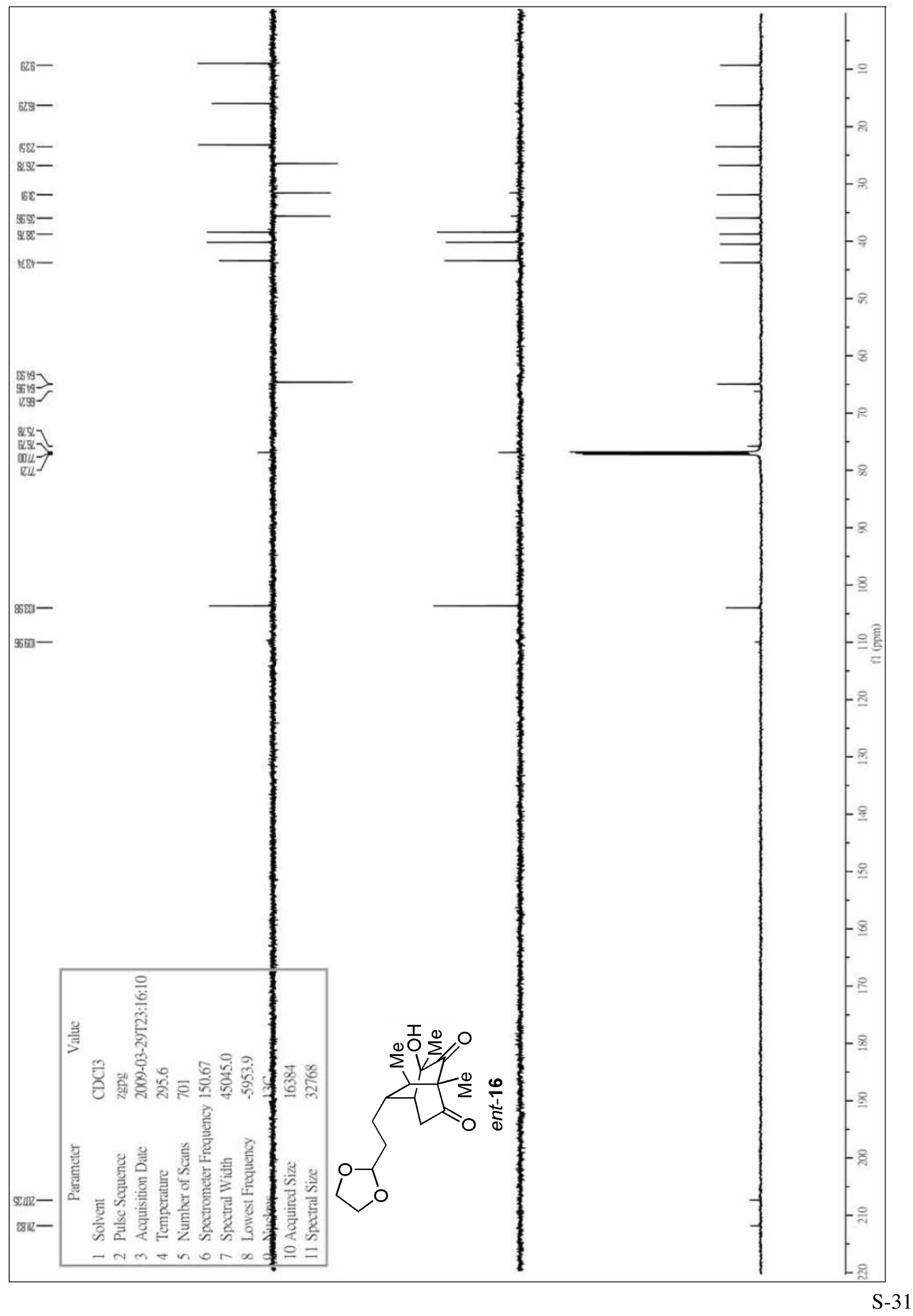




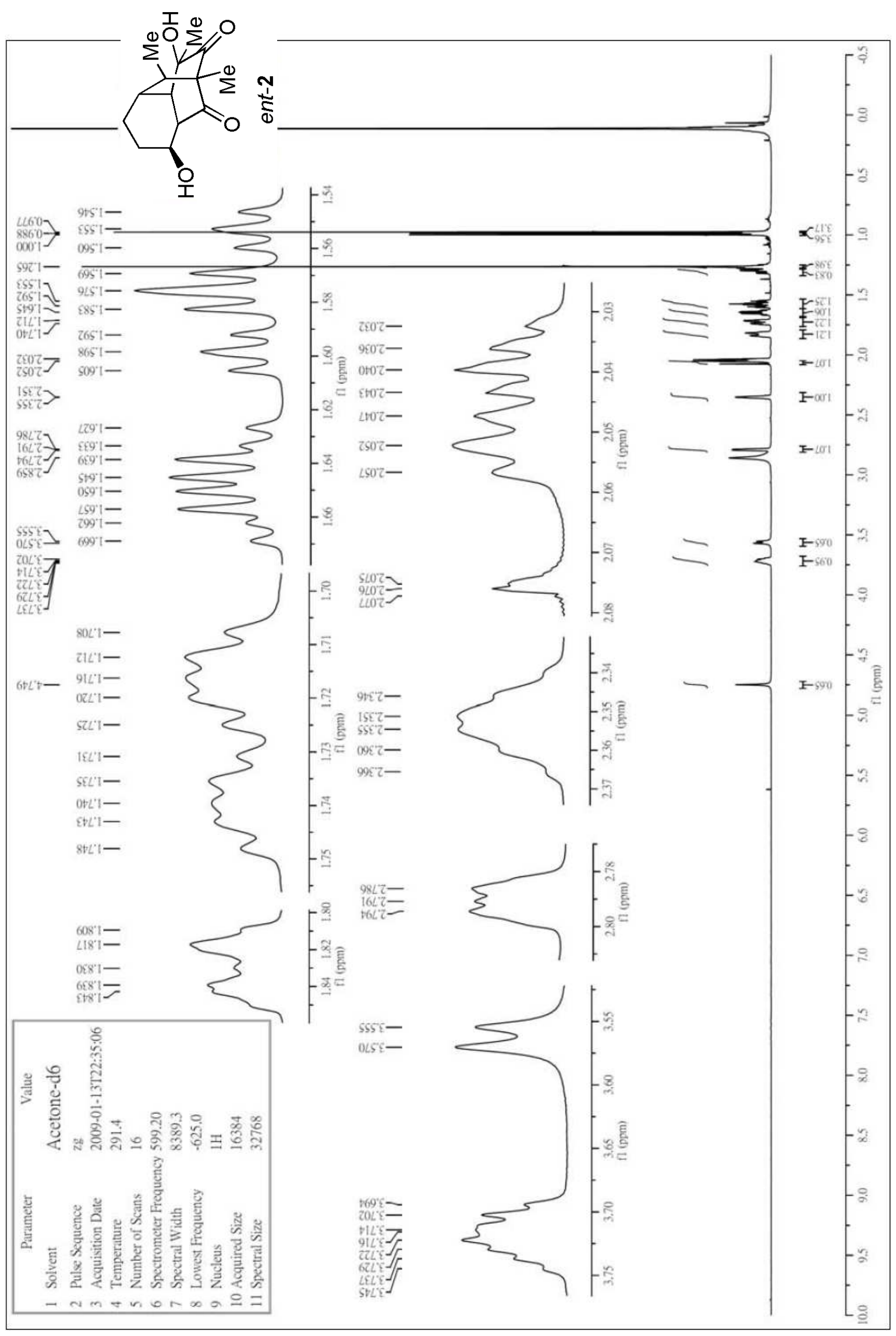




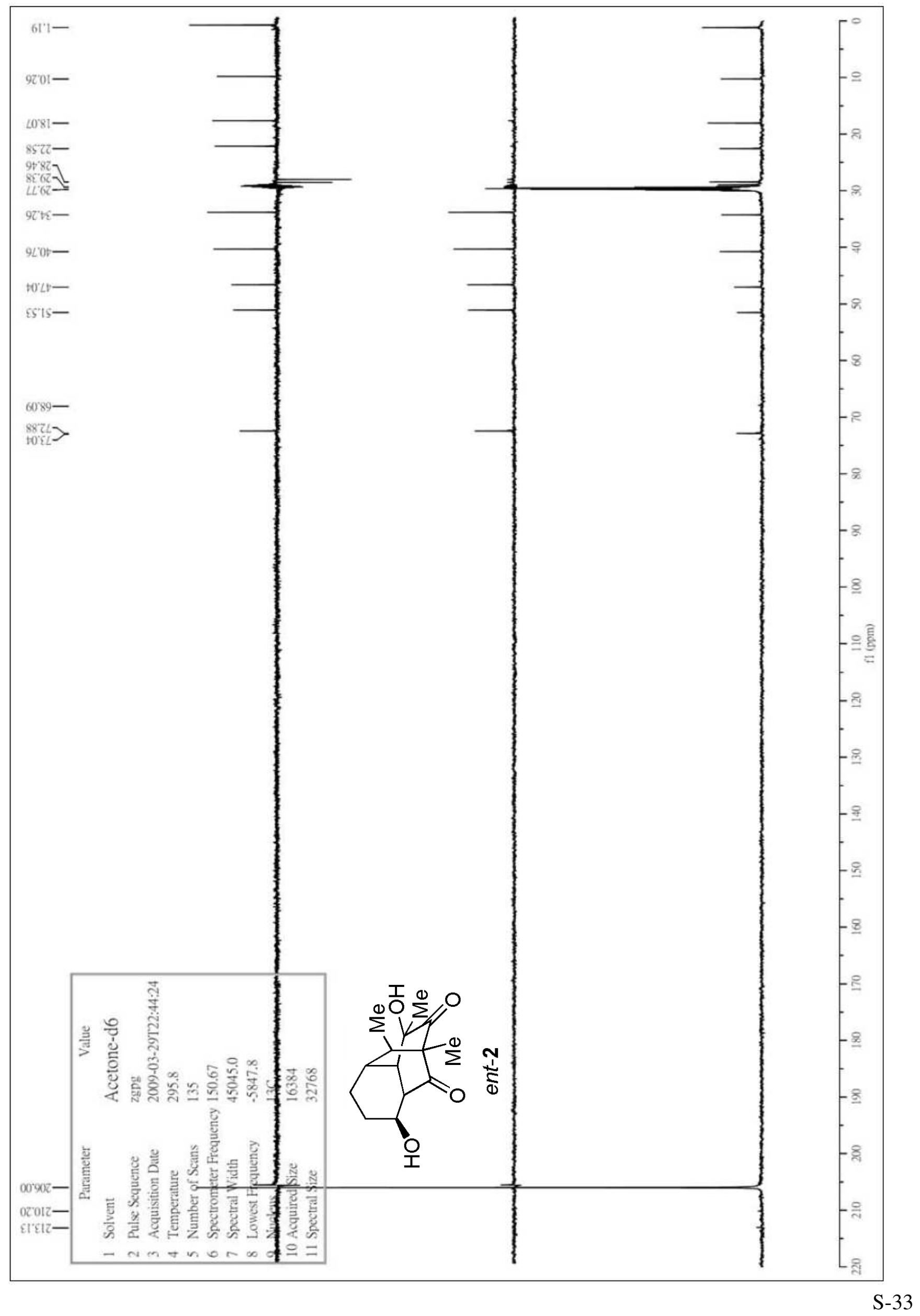




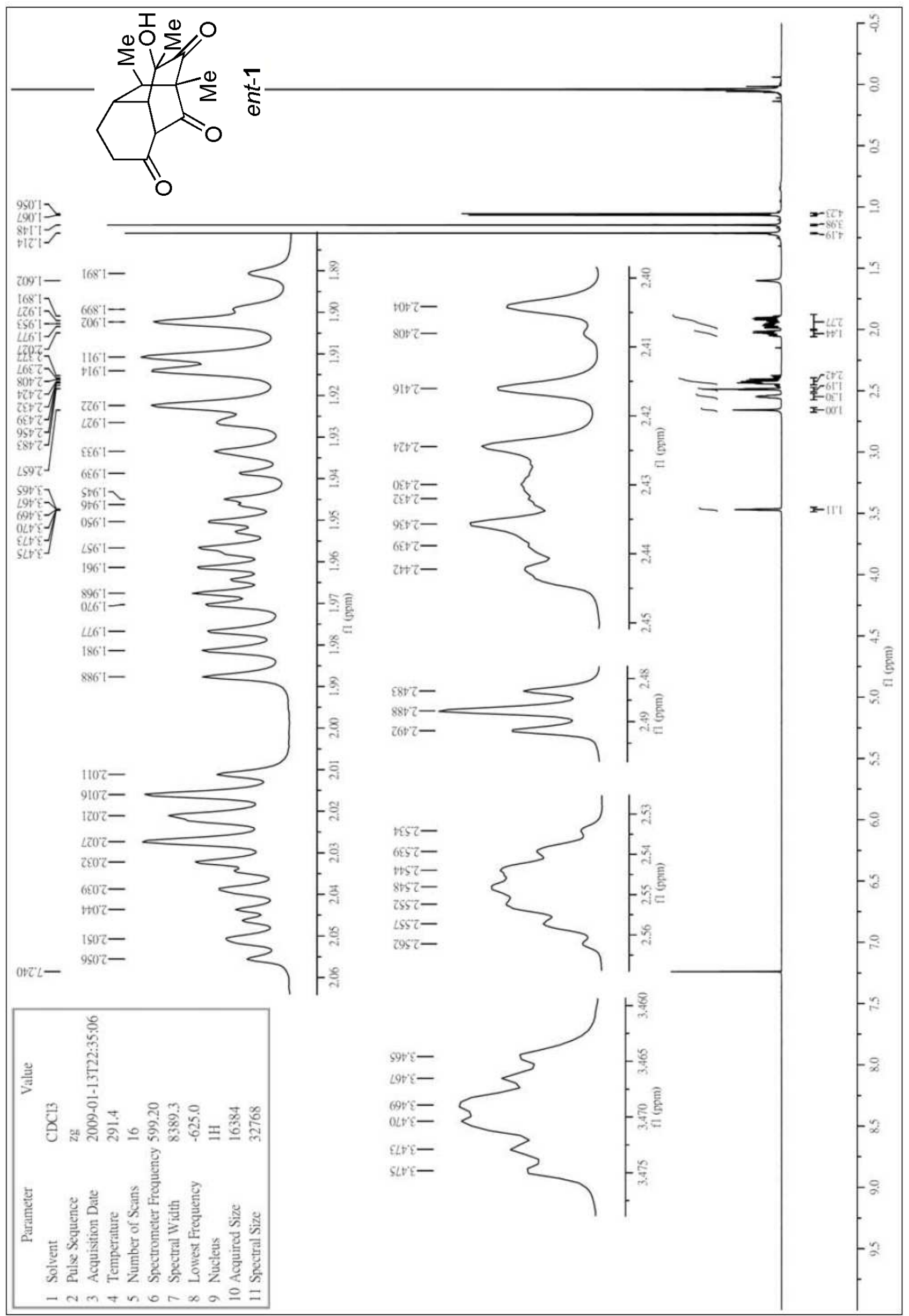




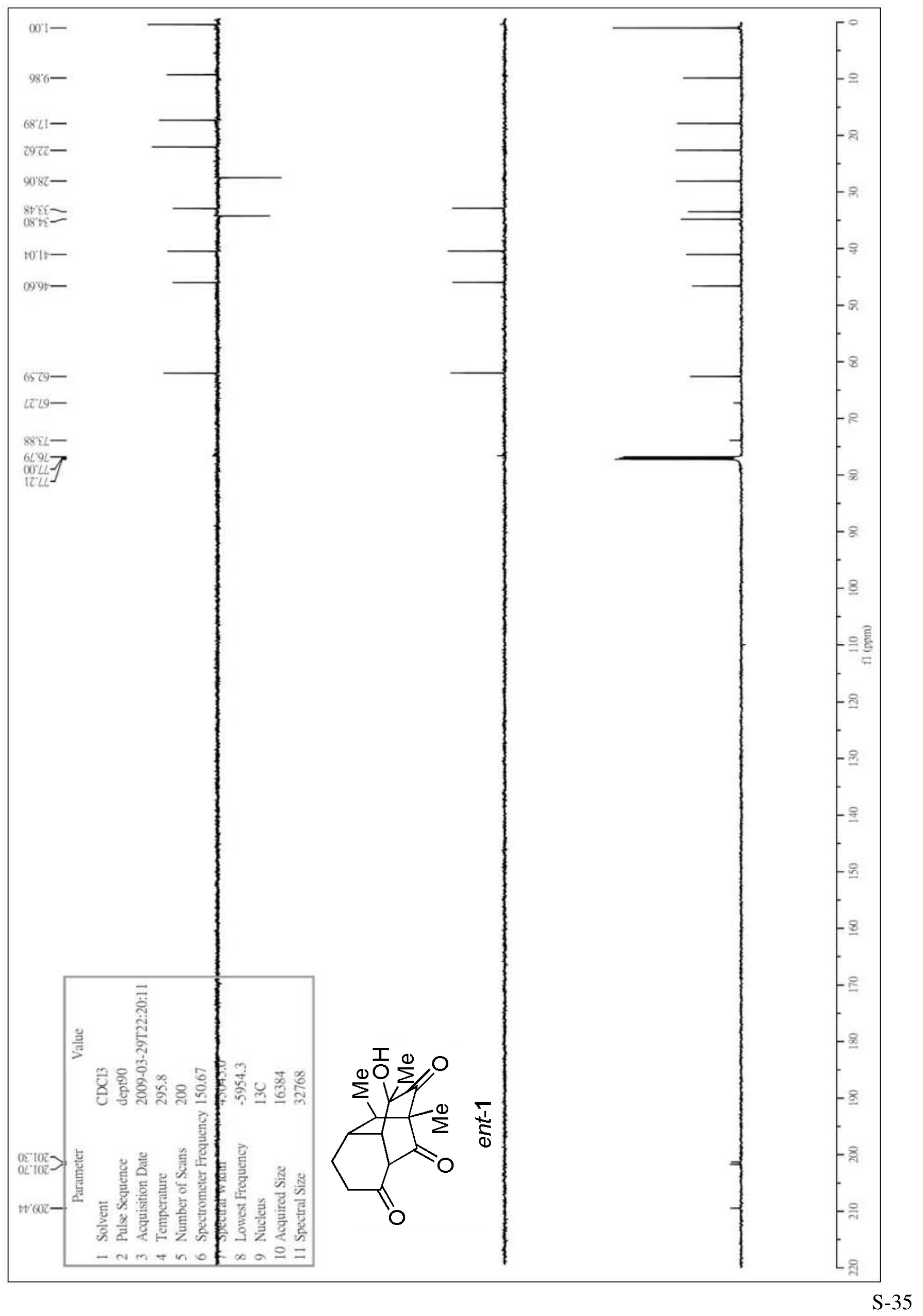




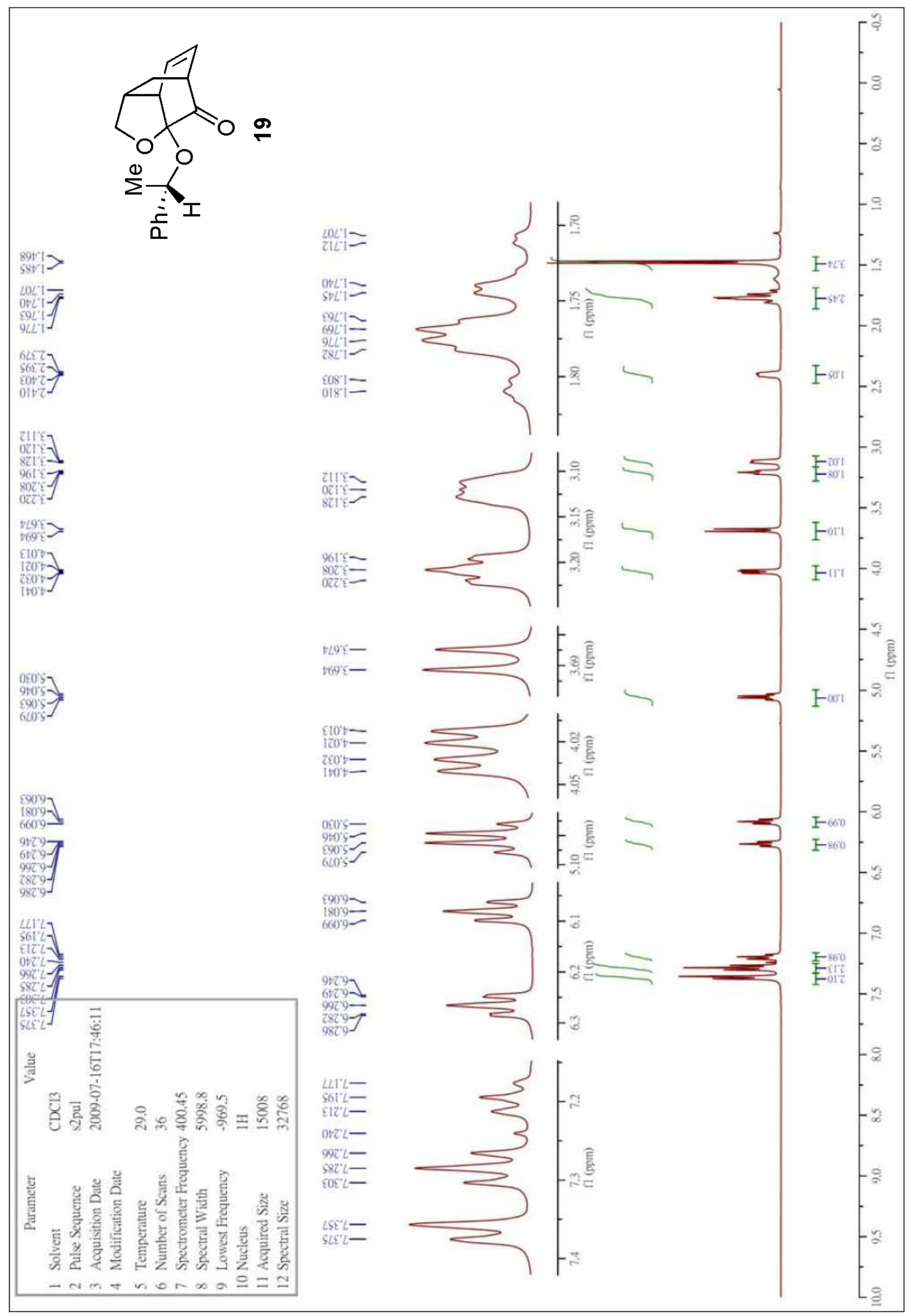




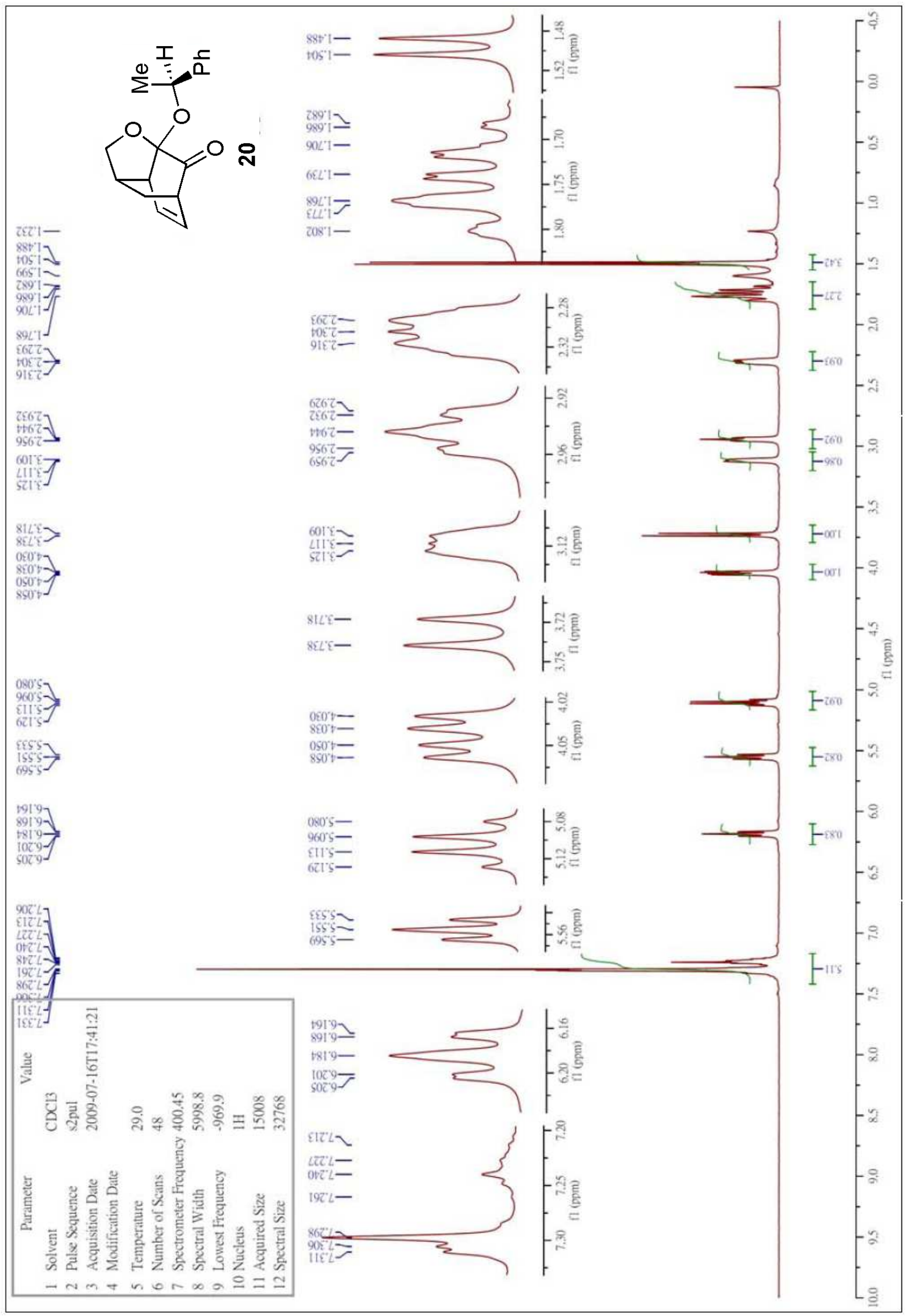




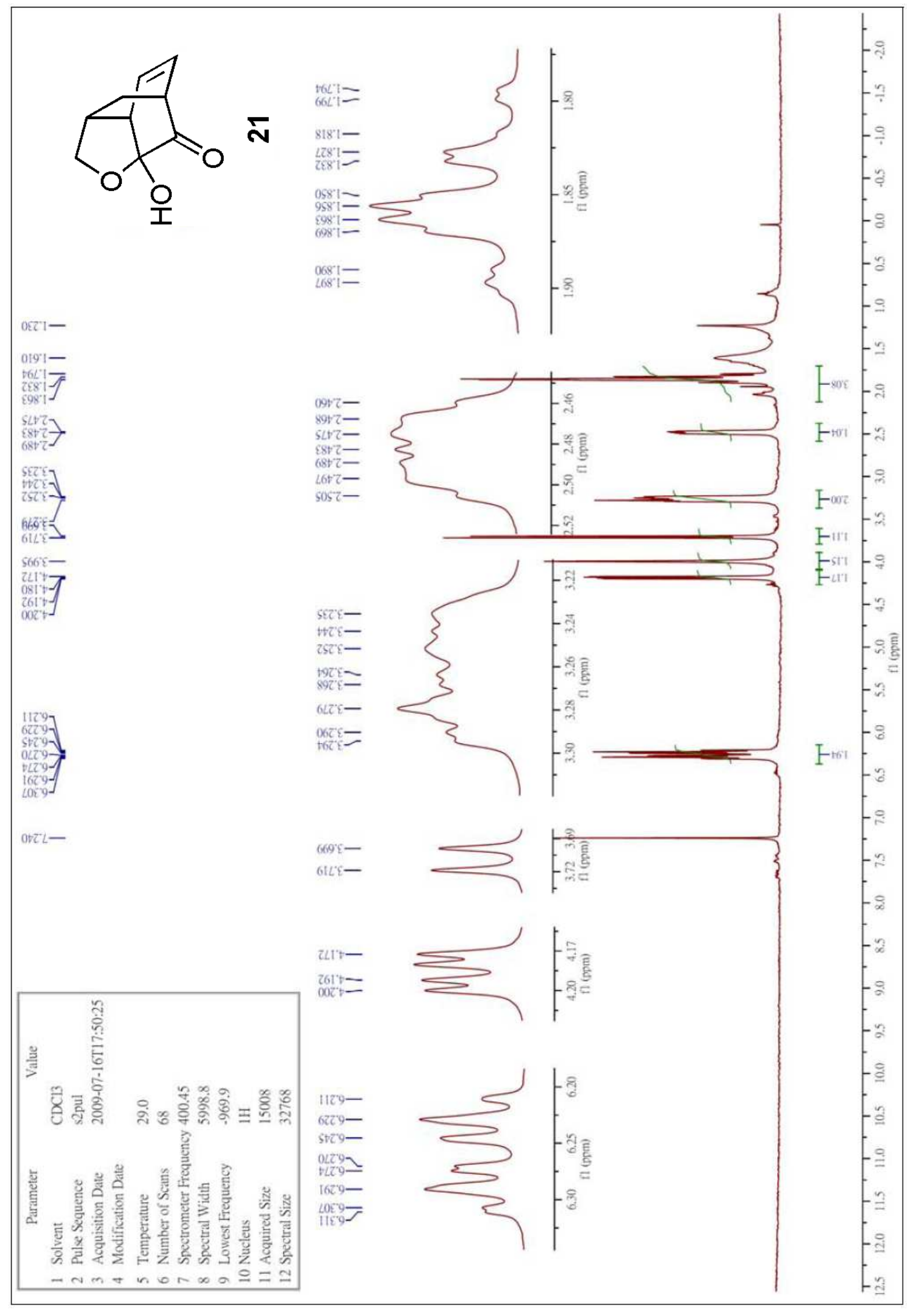




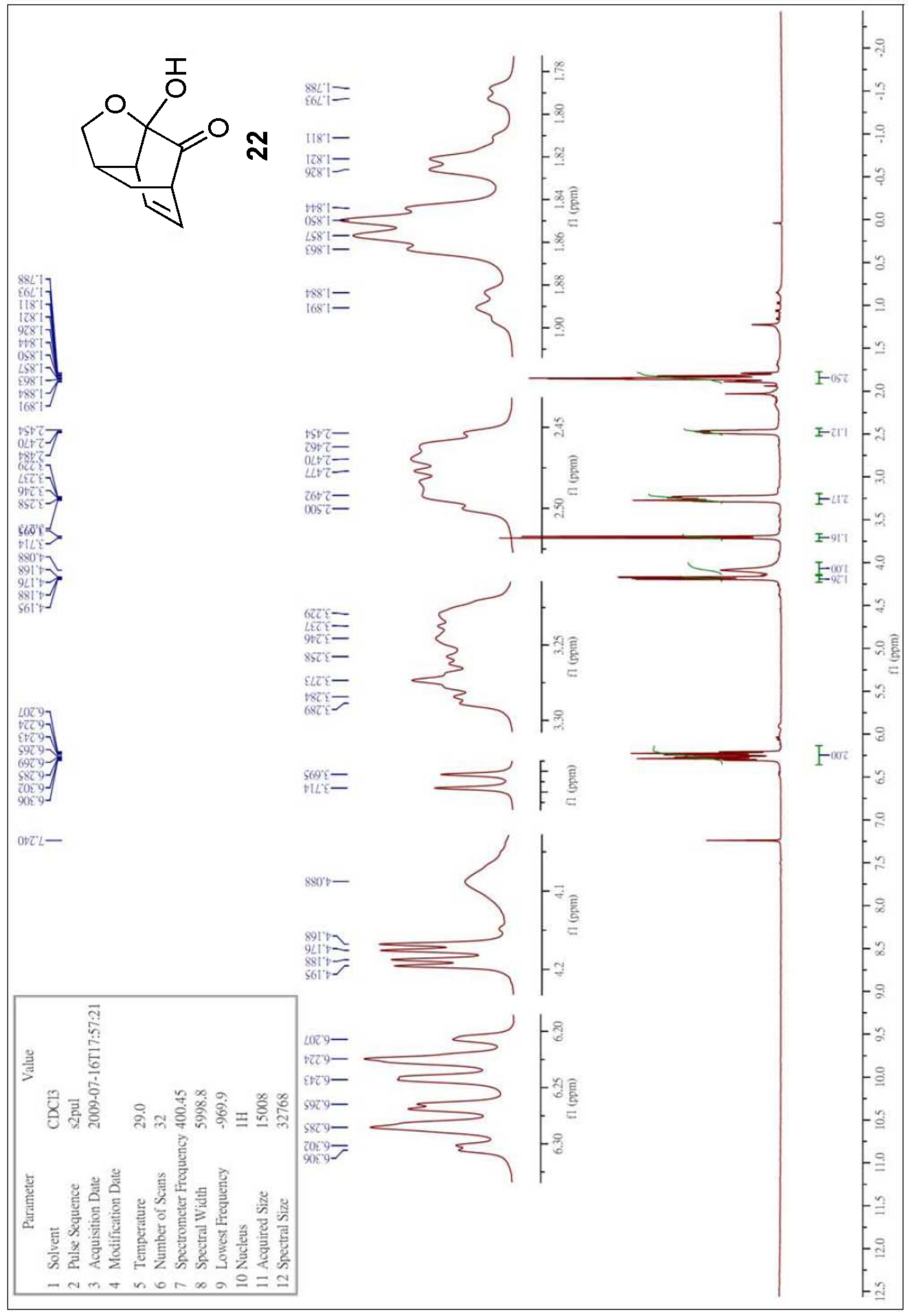

